\title{
Detection and benchmarking of somatic mutations in cancer genomes using RNA-seq data
}

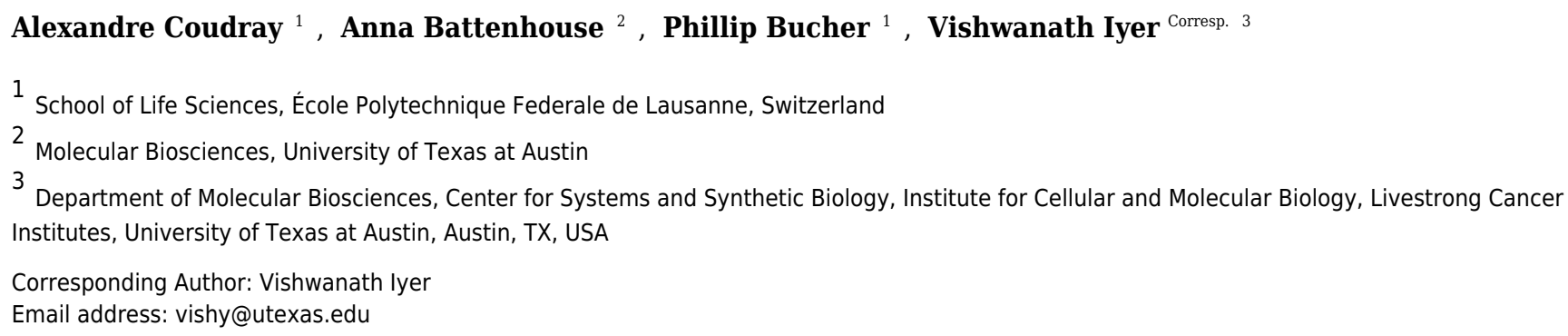

To detect functional somatic mutations in tumor samples, whole-exome sequencing (WES) is often used for its reliability and relative low cost. RNA-seq, while generally used to measure gene expression, can potentially also be used for identification of somatic mutations. However there has been little systematic evaluation of the utility of RNA-seq for identifying somatic mutations. Here, we develop and evaluate a pipeline for processing RNA-seq data from glioblastoma multiforme (GBM) tumors in order to identify somatic mutations. The pipeline entails the use of the STAR aligner 2-pass procedure jointly with MuTect2 from GATK to detect somatic variants. Variants identified from RNA-seq data were evaluated by comparison against the COSMIC and dbSNP databases, and also compared to somatic variants identified by exome sequencing. We also estimated the putative functional impact of coding variants in the most frequently mutated genes in GBM. Interestingly, variants identified by RNA-seq alone showed better representation of GBMrelated mutations cataloged by COSMIC. RNA-seq-only data substantially outperformed the ability of WES to reveal potentially new somatic mutations in known GBM-related pathways, and allowed us to build a high-quality set of somatic mutations common to exome and RNA-seq calls. Using RNA-seq data in parallel with WES data to detect somatic mutations in cancer genomes can thus broaden the scope of discoveries and lend additional support to somatic variants identified by exome sequencing alone. 
Detection and benchmarking of somatic mutations in cancer genomes using RNA-seq data

Alexandre Coudray ${ }^{1}$, Anna M. Battenhouse ${ }^{2}$, Philipp Bucher ${ }^{3}$, Vishwanath R. Iyer ${ }^{2}$

${ }^{1}$ School of Life Sciences, Swiss Federal Institute of Technology (EPFL), CH-1015, Lausanne, Switzerland

${ }^{2}$ Center for Systems \& Synthetic Biology and Department of Molecular Biosciences, The University of Texas at Austin, Austin, TX USA

${ }^{3}$ Swiss Institute for Experimental Cancer Research (ISREC), School of Life Sciences, Swiss Federal Institute of Technology (EPFL), CH-1015, Lausanne, Switzerland

Corresponding Author:

Vishwanath R. Iyer

100 East 24th St. Stop A5000, Austin, TX 78712-1639, USA

Email address: vishy@utexas.edu 


\section{ABSTRACT}

2 To detect functional somatic mutations in tumor samples, whole-exome sequencing (WES) is

3 often used for its reliability and relative low cost. RNA-seq, while generally used to measure

4 gene expression, can potentially also be used for identification of somatic mutations. However

5 there has been little systematic evaluation of the utility of RNA-seq for identifying somatic

6 mutations. Here, we develop and evaluate a pipeline for processing RNA-seq data from

7 glioblastoma multiforme (GBM) tumors in order to identify somatic mutations. The pipeline

8 entails the use of the STAR aligner 2-pass procedure jointly with MuTect2 from GATK to detect

9 somatic variants. Variants identified from RNA-seq data were evaluated by comparison against

10 the COSMIC and dbSNP databases, and also compared to somatic variants identified by exome

11 sequencing. We also estimated the putative functional impact of coding variants in the most

12 frequently mutated genes in GBM. Interestingly, variants identified by RNA-seq alone showed

13 better representation of GBM-related mutations cataloged by COSMIC. RNA-seq-only data

14 substantially outperformed the ability of WES to reveal potentially new somatic mutations in

15 known GBM-related pathways, and allowed us to build a high-quality set of somatic mutations

16 common to exome and RNA-seq calls. Using RNA-seq data in parallel with WES data to detect

17 somatic mutations in cancer genomes can thus broaden the scope of discoveries and lend

18 additional support to somatic variants identified by exome sequencing alone. 


\section{INTRODUCTION}

20 Cancer is among the leading causes of death worldwide, with 8.7 million deaths in 2015 (Global

21 Burden of Disease Cancer Collaboration 2017). As a genetic disease, cancers are driven in part

22 by the accumulation of somatic mutations, which incidentally, also offer targets for new

23 precision therapies directed against tumor-causing mutations (Cancer Genome Atlas Research

24 Network 2013; Yu et al. 2015). Cancer cells typically accumulate somatic alterations that impact

25 specific pathways implicated in cell growth, survival, angiogenesis, motility and other hallmarks

26 of cancer (Hanahan \& Weinberg 2011). Advances in next-generation sequencing technologies

27 have allowed increasingly fast, accurate and cost-efficient analysis of DNA and RNA samples,

28 which has driven the identification of key cancer-driving mutations (Raphael et al. 2014). These

29 findings are beginning to pave the way for new targeted therapies in many cancers, but

30 significant challenges remain (Paez et al. 2004; Taylor et al. 2012).

31 The actual cancer-driving mutations need to be differentiated from somatic passenger mutations

32 caused by impaired DNA repair mechanisms, inherited or de novo germline mutations and

33 neutral polymorphisms, and artefacts that can arise from sequencing errors, PCR or

34 misalignment (Berger et al. 2016; Sahni et al. 2013; Takiar et al. 2017). Moreover, the complex

35 structure of tumors increases the complexity of the analysis, as tumors are typically

36 heterogeneous, containing normal cells as well as distinct clonal lineages of tumor cells

37 (Meacham \& Morrison 2013). Somatic alterations typically range from substitution mutations

38 and small insertions/deletions (indels) to chromosome rearrangements and copy number

39 variations (Rhee et al. 2017).

40 To detect mutations in a tumor sample, whole exome sequencing (WES) has generally been

41 favored over whole genome sequencing (WGS) for its relatively low cost, although dropping 
42 costs of WGS encourage its use for somatic mutation identification (Alioto et al. 2015; Puente et

43 al. 2011). Whole-transcriptome (RNA-seq) data has typically been used to measure gene

44 expression and identify transcript and splicing isoforms. Nevertheless, it is possible to identify

45 genomic variants from RNA-seq (Piskol et al. 2013). Previous studies examining the use of

46 RNA-seq for somatic mutation detection have focused on the characteristics of mutational

47 changes seen in RNA-seq versus WES, but these studies have been limited with regard to cancer

48 type, and there has been little systematic evaluation of the biological novelty and significance of

49 tumor somatic variants detected by RNA-seq (O'Brien et al. 2015).

50 Here, we assessed the utility of RNA-seq for somatic mutation detection in glioblastoma

51 multiforme (GBM), the most common and deadliest form of adult primary brain cancer. GBM

52 shows a median overall survival of only 14-15 months (Stupp et al. 2009). Standard of care for

53 GBM has not changed for many years, and emerging new targeted therapies (mostly targeting

54 angiogenesis-related pathways) unfortunately encounter problems of drug resistance

55 (Stavrovskaya et al. 2016), making the discovery of new target genes of great importance. We

56 focused on the use of the STAR aligner (Dobin et al. 2013) which is fast and is transcript-aware,

57 and therefore has the potential to give additional information about mutations in cancer-activated

58 transcripts that might be missing in WES, and MuTect2 from GATK (Cibulskis et al. 2013)

59 which has been widely used for mutation identification. Our analysis showed that RNA-seq is

60 able to detect novel, GBM-related somatic mutations and can thus complement exome and

61 whole-genome sequencing in identifying somatic mutations in tumor genomes. 


\section{MATERIALS AND METHODS}

\section{Methods overview, sample preparation, data origin and databases used}

64 We developed a new pipeline to detect somatic mutations in RNA-seq data, combining RNA-seq

65 alignment using a STAR 2-pass procedure with somatic mutation detection using MuTect2 for

66 variant calling (Cibulskis et al. 2013). Variants from RNA-seq and WES were compared, first,

67 on a pair of RNA-seq/WES from a GBM tumor that had already been analyzed in our laboratory

68 (Hall et al. 2018) and then on a set of 9 pairs of RNA-seq and WES data from GBM tumors

69 analyzed by the Cancer Genome Atlas (TCGA) (Brennan et al. 2013). We compared and

70 evaluated RNA-seq and WES mutations in four steps. First, we estimated the proportion of

71 germline or somatic mutations by comparison of identified variants to the dbSNP database (Kitts

72 et al. 2013) which catalogs known germline variants, and the Catalogue Of Somatic Mutations in

73 Cancer or COSMIC database (Forbes et al. 2015) respectively. The use of these databases

74 allowed us to evaluate whether a variant was a germline (included in dbSNP but not in

75 COSMIC) or a somatic mutation (included in COSMIC but not in dbSNP). Second, somatic

76 mutations detected in RNA-seq-only data were consolidated to highlight mutations present in

77 multiple tumor samples. Third, their functional impact on proteins was evaluated by using two

78 scoring systems: SIFT and FATHMM with cancer-weights (FATHMMcw) (Ng \& Henikoff

79 2003; Shihab et al. 2013b). Finally, we focused on mutations affecting a set of 29 genes already

80 shown to be implicated in GBM by a previous TCGA study (Cancer Genome Atlas Research

81 Network 2008). Mutations falling into coding regions of these 29 genes and showing high

82 likelihood of altered protein function were assumed to be the best GBM-related mutations and

83 potential cancer-drivers. Finally, we repeated this analysis on an independent validation dataset

84 consisting of 15 pairs of RNA-seq and WES data from TCGA. 
85 We generated paired RNA-seq and WES data from one GBM tumor (SD01) collected at St.

86 David's Medical Center (Austin, TX) after written informed consent, in a study approved by the

87 Institutional Review Boards of St. David's Medical Center and of the University of Texas at

88 Austin (approval numbers AMIRB 10-5-03 and 2012-01-0040). For WES and RNA-seq, we

89 used the exome capture kit NimbleGen SeqCap EZ (Roche) and the NEBNext small RNA kit

90 (NEB) respectively. Sequencing was carried out at the NGS Core Facility of the MD Anderson

91 Cancer Center Science Park on an Illumina HiSeq 2500. Data is available in dbGaP

92 (https://www.ncbi.nlm.nih.gov/projects/gap/cgi-bin/study.cgi?study_id=phs001389.v1.p1). For

93 GBM data from TCGA, BAM files resulting from alignment were downloaded from the

94 Genomic Data Commons data portal and used directly in the subsequent analysis pipeline since

95 they were already aligned with STAR. To evaluate variants, two databases were used: dbSNP

96 (Kitts et al. 2013) with the b147 build on the GRCh38 reference $\left(37 \times 10^{6}\right.$ variants), and the

97 COSMIC database v78 (Forbes et al. 2015), which contains $3.3 \times 10^{6}$ known somatic variants.

98 We carried out all analyses using the GRCh38 primary assembly reference acquired from

99 GENCODE (Harrow et al. 2012). ANNOVAR (v.2016Feb01) (Wang et al. 2010) was used to

100 annotate variants relative to RefSeq annotations (release 73) (O'Leary et al. 2016).

101 A pipeline to detect variants from RNA-seq data with STAR 2-pass and GATK MuTect2 102 and distinguish GBM-related mutations

103 The general pipeline used is shown in Fig. 1, with slight differences between samples (SD01 and

104 TCGA) or techniques (RNA-seq and WES) as depicted in Fig. 1B. The workflow was adapted

105 from GATK best practices for variant calling (Van der Auwera 2014; Van der Auwera et al.

106 2013) but using MuTect2 for variant calling. The process first involved trimming the adapters

107 with cutadapt (v1.10) (Martin 2011) from fastq files, removing sequences that were shorter than 
10836 bases after trimming, and removing rRNA and tRNA sequences by aligning with BWA

109 (v0.7.12-r1039) (Li \& Durbin 2009) to a reference built with known rRNA/tRNA. Filtered reads

110 were then aligned with STAR aligner (v2.4.2a) using a 2-pass procedure (Dobin \& Gingeras

111 2015). Before variant calling, aligned reads in BAM format were sorted, duplicate reads were

112 flagged (MarkDuplicates, Picard v2.5.0), the base scores recalibrated (BaseRecalibrator, GATK

113 v3.6) and RNA-seq reads were split into exons (SplitNCigarReads, GATK v3.6). Variant calling

114 was done with MuTect2 in tumor versus normal mode as described below. Variants recovered in

115 VCF files were then separated into RNA-seq-only, Intersection and WES-only. ANNOVAR

116 (v.2016Feb01) (Wang et al. 2010) was used to annotate variants relative to RefSeq annotations

117 (release 73) (O'Leary et al. 2016). SIFT score/prediction (v2.3) (Ng \& Henikoff 2003), and

118 FATHMM score/prediction with cancer weights (v2.3) (Shihab et al. 2013a; Shihab et al. 2013b)

119 were used to evaluate the functional impact of non-synonymous SNVs and frameshift indels.

120 Finally, a set of 29 genes known to be related to GBM (Cancer Genome Atlas Research Network

121 2008) was used to evaluate GBM-related mutations in specific pathways.

\section{Variant Calling Using MuTect2 from Genome Analysis ToolKit (GATK)}

123 MuTect2 infers genotypes with two log-odd ratios (Cibulskis et al. 2013) which score the

124 confidence that a mutation is present in the tumor sample (TLOD score) and is absent from the

125 matched-normal sample (NLOD score). The thresholds used by MuTect2 to consider a variant as

126 being real and somatic (leading to the annotation "PASS") are by default TLOD $>6.3$ and

127 NLOD $>2.2$. For dbSNP variants, a higher NLOD threshold of 5.5 is used, except if the variant

128 is also present in the COSMIC database. 


\section{Building a Panel of Normals (PoN) for variant calling with MuTect2}

130 The creation of a PoN is an optional step that improves variant calling by filtering out method-

131 specific artefacts, by doing variant calling (MuTect2) on a set of normal samples (Fig. 1A). The

132 samples for the PoN should ideally be obtained through protocols and data processing steps

133 closely matched to the tumor sample. For this reason, two PoN were built, one with RNA-seq

134 data from normal samples and another with WES data from normal samples. Then, variants

135 identified by MuTect2 in at least two normal samples were compiled together into one PoN VCF

136 file. Although using 30 normal samples is recommended by GATK, we used only 12 normal

137 samples as they were matched to the 12 GBM tumor samples from TCGA.

\section{MuTect2 filters}

139 Based on the TLOD score, MuTect2 will reject a variant when a specific TLOD $>6.3$ threshold

140 is not reached, suggesting insufficient evidence of its presence in the tumor sample $\left(t \_l o d \_\right.$star

141 filter). homologous_mapping_event is a filter that detects homologous sequences and filters out

142 variants falling into sequences that have 3 or more events observed in the tumor.

143 clustered_events is a filter for clustered artifacts. str_contraction filters out variants from short

144 tandem repeat regions. alt_allele_in_normal filters out variants if enough evidence is shown of

145 its presence in the normal sample (NLOD threshold > 2.0). multi_event_altallele_in_normal

146 filters out a variant when multiple events are detected at the same position in the matched-normal

147 sample. germline_risk filters out variants that show sufficient evidence of being germline based

148 on dbSNP, COSMIC and the matched-normal sample (NLOD value). panel_of_normals filters

149 out variants present in at least two samples of the panel of normals. 


\section{RefSeq annotations with ANNOVAR}

151 ANNOVAR (v.2016Feb01) (Wang et al. 2010) was used to annotate the variants in the VCF file

152 with RefSeq Genes annotations (release 73 with reference GRCh38) (O'Leary et al. 2016) and

153 SIFT scores/predictions (v2.3) (Ng \& Henikoff 2003). RefSeq gives the closest gene name, or

154 the two closest genes whenever a variant falls within intergenic regions. RefSeq also gives

155 information about the type of mutation and the eventual amino acid change, whenever a variant

156 falls in a coding region. For effects on alternative splicing, RefSeq gives a list of all possible

157 transcripts.

\section{Scoring non-synonymous SNVs and indels with SIFT score}

159 One way to assess the functional impact of an amino-acid (AA) change is to use SIFT (Ng \&

160 Henikoff 2003), which uses homologous sequence comparison. SIFT (v2.3) gives a score based

161 on the frequency at which an AA appears at a specific location in functionally related protein

162 sequences. The AA change is given a predicted score: Tolerated $(p>0.05)$ or Deleterious $(p<$

163 0.05). Low scores typically occur in highly conserved regions that tend to be intolerant to most

164 substitutions. On the contrary, unconserved regions tend to be more tolerant to AA changes.

165 SIFT indel has been developed for scoring frameshifting indels (Hu \& $\mathrm{Ng} \mathrm{2013),} \mathrm{which} \mathrm{relies} \mathrm{on}$

166 a different algorithm based on a machine learning model. It gives a prediction of damaging or

167 neutral along with a confidence score.

\section{Scoring non-synonymous SNVs and indels with FATHMM cancer-weighted scores}

169 Functional Analysis through Hidden Markov Models (FATHMM v2.3) also uses homologous

170 protein sequences to find the probability of an amino acid substitution at a given position. The

171 algorithm relies on Hidden Markov models to compute probabilities, its final scores being a ratio

172 between the probability of the wild-type and the mutant AA. The version used here (Shihab et al. 
173 2013b) also incorporates cancer weights (FATHMMcw), the frequency of cancer-associated

174 variants from the CanProVar database and wild type weights, the frequency of neutral

175 polymorphisms from UniRef database falling in the same protein region as the variants. The final

176 score is an indication whether an AA substitution is deleterious and associated with cancer

177 (prediction CANCER given for score < -0.75) or neutral (prediction PASSENGER given for

178 score $>-0.75$ ). FATHMM for indels (Shihab et al. 2015) works on indels shorter than $20 \mathrm{bp}$ and

179 emits a prediction (pathogenic or neutral) together with a confidence score (expressed in \%).

180 Criterion to build a set of 29 genes previously shown to be altered in GBM

181 A set of 29 genes that were shown to be the most frequently mutated genes in GBM by a TCGA

182 study on 91 GBM samples (Cancer Genome Atlas Research Network 2008) was used to look for

183 somatic mutations in GBM-related pathways. Genes selected to be part of the set were ARF,

184 BRCA2, CBL, CDK4, CDKN2B, CDKN2C, EGFR, EP300, ERBB2, ERBB3, FGFR2, IRS1,

185 MDM2, MDM4, MET, MSH6, NF1, P16, PDGFRB, PIK3C2B, PIK3C2G, PIK3CA, PIK3R1,

186 PRKCZ, PTEN, RB1, SPRY2, TP53 and TSC2. These genes were shown to bear mutations in at

187 least $2 \%$ of samples, the most altered being ARF (49\%), EGFR (45\%), PTEN (36\%) and TP53

188 (35\%). The "Best GBM-related mutation" (Table 1) is indicated when a mutation was included

189 in this set of 29 genes, part of COSMIC database but not in dbSNP, resulted in an AA change

190 and retained based on both SIFT and FATHMM scores as being functionally deleterious for

191 protein function. 


\section{RESULTS}

193 Read counts and variant features highlight differences between RNA-seq and WES variants 194 in TCGA samples

195 In the majority of samples, RNA-seq showed fewer uniquely mapped reads than WES (Fig. 2A 196 and Supplementary Fig. S1). Secondary alignments and unmapped reads were generally higher

197 in the RNA-seq data, which could be due in part to unmapped splice junction reads and

198 mismatches in RNA-seq due to RNA editing. Adenosine to inosine (A-to-I) is the most common

199 form of RNA editing in humans, leading mainly to $\mathrm{A}>\mathrm{G}$ and $\mathrm{T}>\mathrm{C}$ base substitutions (Picardi et

200 al. 2015), which were clearly enriched in RNA-seq compared to WES data (Fig. 2B). RNA

201 editing site databases like DARNED (Kiran et al. 2013), RADAR (Ramaswami \& Li 2014) or

202 Inosinome Atlas (Picardi et al. 2015) could potentially be used to filter out such variants (Piskol

203 et al. 2013).

204 The proportion of variants filtered by the different MuTect2 filters are shown in Fig. 2C.

205 MuTect2 generates two log-odd ratios, TLOD and NLOD, which can be used to infer the

206 somatic origin of a variant (Materials and Methods). RNA-seq variants showed lower TLOD

207 scores and slightly higher NLOD scores than WES variants. Low read counts or poor base

208 qualities supporting the altered allele in tumor can lead to low TLOD values. Fewer RNA-seq

209 variants met the TLOD threshold (Fig. 2C, TLODfstar). Interestingly, TLOD scores of COSMIC

210 variants were higher than non-COSMIC variants (Supplementary Fig. S2), suggesting that

211 TLOD reflects the higher true positive rate. On the other hand, variants that also occur in the

212 matched-normal samples could be filtered by the AltAlleleInNormal MuTect2 filter based on

213 NLOD values. RNA-seq data from TCGA samples showed particularly low numbers of variants

214 excluded by this filter (Fig. 2C), which could be due to coverage differences between tumor 
215 RNA-seq and matched-normal (the latter being WES data). Thus, distinct variant features given

216 as an output by MuTect2 could be used to build a variant filtering model (Ding et al. 2012).

217 Variants accepted as true and somatic $(P A S S)$ by MuTect2 were higher in RNA-seq than WES

218 for all TCGA samples (Fig. 2D). The overlap between RNA-seq and WES was small in all

219 samples, but interestingly, the overlap increased with increasing significance of the variants. An

220 average of only $6.60 \%$ of WES variants retained by MuTect2 (PASS) were also present in RNA-

221 seq, while $15.9 \%$ of WES variants from coding regions and $17.2 \%$ of functional mutations were

222 common to RNA-seq (Fig. 2D). Coverage differences between RNA-seq and WES could

223 partially explain the phenomenon. A previous study indeed found that $~ 71 \%$ of RNA-seq

224 variants fell outside the WES capture boundaries (O'Brien et al. 2015). Moreover, they showed

225 that a high proportion of RNA-seq-only variants were missed by WES because of their low allele

226 fraction (AF).

227 As expected, the RNA-seq/WES intersection was enriched in variants from coding regions

228 (89.7\% of coding variants), since both RNA-seq and WES query exons. RNA-seq data also

229 showed an unexpected level of intronic/intergenic variants. Intronic mRNA reads could partly

230 come from unspliced RNA (pre-mRNA). A previous study has indeed detected many intronic

231 mRNA variants, which could come from inefficient splicing in cancer (Sowalsky et al. 2015). On

232 the other hand, intergenic RNA-seq variants could come from unannotated genes, non-coding

233 RNA, retrotransposons, splicing errors (Pickrell et al. 2010) and sequencing/mapping errors.

\section{Allele fraction and coverage are useful features to further classify variants}

235 In theory, heterozygous mutations would show an allele fraction (AF) around 0.5. However,

236 somatic mutations from cancer cells are expected to appear at lower frequencies, as tumor 
237 samples are heterogeneous and not pure clones. Moreover, copy number variation (CNVs) can

238 lead to gain/loss of chromosomes and/or duplications of genes (Yin et al. 2009). RNA-seq-only

239 variants showed a surprising $\mathrm{AF}$ distribution in that $38.2 \%$ showed an $\mathrm{AF}>0.95$ (Fig. 3A,B)

240 versus only $0.50 \%$ of WES-only variants. These high AF RNA-seq-only variants showed low

241 coverage, and the majority of them occurred in intronic/intergenic regions (85.6\% of RNA-seq-

242 only variants with AF $>0.95$ ). Conversely, we also found a high number of RNA-seq-only

243 variants showing $\mathrm{AF}<0.05$ (36.3\% of RNA-seq variants representing 4267 variants in nine

244 TCGA samples). In comparison, WES-only data showed only 502 variants (22.8\%) with AF $<$

245 0.05. Low AF RNA-seq-only variants mainly originated from coding regions (81.1\% of RNA-

246 seq-only variants with $\mathrm{AF}<0.05$ ) and often showed high coverage, which distinguished them

247 from WES-only variants (Fig. 3A). We examined the coverage data for RNA-seq-only variants

248 with $\mathrm{AF}<0.05$ and coverage $>500$, and found only one variant (out of 2192) that was also

249 present in WES data, and showing only one altered read. This region of high coverage/low AF is

250 of particular interest as it is likely to contain true somatic mutations that are missed in WES data.

251 COSMIC/dbSNP overlap can be used as an indicator of the somatic/germline content in

252 TCGA samples

253 For each of the three classes of variants - WES-only, Intersection and RNA-seq-only, we

254 examined the proportion in different genomic regions (Fig. 3C), potential for affecting protein

255 function (Fig. 3D) and representation in dbSNP and COSMIC databases (Fig. 3E). The

256 proportion of variants included in the dbSNP database is potentially an indicator of germline

257 content among identified variants, while the overlap with the COSMIC database can serve as an

258 indicator of somatic mutations (Fig. 3E). It must be noted that with the increasing coverage in

$259 \mathrm{dbSNP}$ of variants from ever-increasing numbers of human genomes, inclusion in dbSNP cannot 
260 always rule out a somatic variant (Nadarajah et al. 2016). Nevertheless, their overlap is small, at

261 least in the versions of the databases we used, with only $0.15 \%$ of dbSNP variants included in

262 COSMIC (Supplementary Fig. S3). Coding variants identified by both RNA-seq \& WES

263 (Intersection) showed a particularly high proportion (87.7\%) included in COSMIC but not in

264 dbSNP (COSMIC-only), which may be considered the most likely candidates for somatic

265 mutations. A high proportion of WES-only coding variants (39.5\%) and a low proportion of

266 RNA-seq-only coding variants (3.0\%) were likewise found in COSMIC-only but although the

267 proportions were very different, both WES-only and RNA-seq-only variants contained the same

268 order-of-magnitude COSMIC-only variants (Fig 3E). Thus, RNA-seq-only identified 138

269 COSMIC-only variants from coding regions that were therefore missed by WES-only. Because

270 COSMIC contains variants discovered mainly by WES, it is possible that many of the RNA-seq-

271 only variants unknown to both COSMIC and dbSNP, representing 96.4\% of RNA-seq-only

272 variants from coding regions (4402 variants in 9 samples), could include many bonafide cancer

273 somatic mutations. We therefore explored this possibility further.

\section{Genes showing somatic mutations in multiple TCGA samples only in RNA-seq data}

275 There were 63 genes with RNA-seq-only variants that were mutated in 5 or more tumors, and

276 many genes from this group have been implicated in cancer (Fig. 4). For example, a set of three

277 complement related genes - complement C3, $\alpha-2$ macroglobulin (A2M) and the complement

278 lysis inhibitor SP-40/clusterin (CLU) - that have been implicated in various cancers including

279 gliomas (Reis et al. 2018; Saratsis et al. 2014; Shinoura et al. 1994; Suman et al. 2016) were

280 present in this group, and interestingly, these three proteins have been recently shown to form a

281 network of related biomarkers in B-ALL (Cavalcante Mde et al. 2016). One tumor contained a

282 cluster of highly mutated genes (Fig. 4, bottom left), including SPARC and FLNA, which are 
283 associated with cell-matrix interactions and cell motility (Neuzillet et al. 2013; Xu et al. 2010),

284 and thus possibly involved in metastasis. On the other hand, MAGED1 was linked with cell-

285 death mechanisms (Mouri et al. 2013), which are often disrupted in cancer. One frameshift

286 insertion was detected in the ARF1 gene located at the exact same position (G14fs) in all nine

287 samples. This was a COSMIC-only variant with plausible AF and coverage. Despite high

288 coverages in WES at the variant position, the insertion was never present in WES data, and since

289 insertions/deletions have been shown to be more prone to artefacts (Kroigard et al. 2016), it was

290 not retained in Table 1 and 2 (see below). Note that the mutational landscape presented here is

291 distinct from the one obtained by a TCGA study on WES data (Brennan et al. 2013), which is not

292 surprising as RNA-seq-only data is likely interrogating other regions of the genome relative to

293 WES.

294 Analysis of somatic mutations found by RNA-seq without a corresponding matched normal 295 sample

296 The SD01 GBM tumor sample had no corresponding matched normal to enable reliable

297 distinction of somatic mutations from germline variants, so it presented unusual challenges.

298 However, it is worthwhile to consider such samples because often, RNA-seq data may be

299 available from a tumor without a corresponding matched normal sample. The total number of

300 variant called in SD01 was much higher than the average TCGA sample (by 10.5-fold for RNA-

301 seq and 17.7-fold for WES). SD01 had a similar number of aligned reads as the TCGA samples

302 for both RNA-seq and WES, so the higher number of somatic variants could be in part due to the

303 absence of matched-normal, the small panel of normals used and/or by a higher underlying

304 mutation rate in this specific tumor. MuTect2 variant calling was carried out in tumor-only mode

305 and only relied on TLOD values without distinction between somatic and germline variants. 
306 Many dbSNP variants were indeed observed (Supplementary Fig. S4). The distribution of SD01

307 variants by chromosome showed a remarkably high number of variants on Chromosome 7

308 (Supplementary Fig. S5), which could reflect amplification of Chromosome 7, a common feature

309 in GBM (Cancer Genome Atlas Research Network 2008). SD01 also showed a higher density of

310 transition variants $(\mathrm{T}>\mathrm{C}, \mathrm{C}>\mathrm{T}, \mathrm{A}>\mathrm{G}$ and $\mathrm{G}>\mathrm{A})$, which tend to be less deleterious, as expected for

311 germline variants (Campbell \& Eichler 2013). Nevertheless, SD01 RNA-seq-only variants

312 included several interesting candidate somatic mutations. One of these RNA-seq-only mutations

313 was EGFR-A702S, found in COSMIC but not in dbSNP, and retained by both SIFT and

314 FATHMM scores (see below). Two other frameshift insertions were also found by RNA-seq-

315 only data in EGFR (S229fs and W477fs), with COSMIC variants found at the same amino-acid

316 coordinates (Table 2). Moreover, the intersection between RNA-seq and WES data in SD01

317 showed other interesting candidates, such as a point mutation in EGFR (A289V - retained by

318 both SIFT/FATHMM, and present in COSMIC but not in dbSNP).

319 Analyzing the functional impact of somatic mutations on protein function in relation to cancer and GBM pathways

321 We used the algorithms FATHMM and SIFT to evaluate the potential impact of somatic variants

322 on protein function in cancer pathways (Materials and Methods). The FATHMM and SIFT score

323 distributions showed a significant difference only for FATHMM scores between the Intersection

324 and WES-only (Fig. 5). Many RNA-seq-only variants scored below both FATHMM and SIFT

325 thresholds, indicating they could be potential functional mutations. The overall proportion of

326 variants retained by FATHMM and SIFT was higher for Intersection variants (11.8\%, Fig. 5D),

327 and slightly higher in RNA-seq-only than WES-only. Mutations present among a set of 29 hand-

328 curated GBM-related genes were designated as the "best GBM-related mutations" (Table 1), and 
329 comprised 11 mutations. RNA-seq-only detected 3 of these 11 mutations, while WES-only found

3302 and the Intersection between RNA-seq and WES found 6 of the 11 GBM mutations. These

331 three RNA-seq-only mutations (EGFR-A702S, TP53-I254S and TSC2-V296fs) are thus cancer-

332 driver candidates found only by RNA-seq and should therefore motivate the use of RNA-seq as

333 they were missed by WES. Taken together, our results suggest that the intersection between

334 RNA-seq data and WES yielded the highest quality GBM-related mutations in TCGA samples,

335 for 3 reasons. First, variants from RNA-seq/WES intersection showed 90.5\% of COSMIC-only

336 variants (Fig. 3E), an average much higher than WES-only or RNA-seq-only data. Second,

337 coding variants from the intersection also showed more evidence of functional alteration through

338 their SIFT and FATHMM scores (Fig. 5). Third, 6/11 of the "best GBM-related mutations" were

339 identified in the intersection (Table 1), even though it was the smallest group in term of variant

340 number. Thus combining RNA-seq and WES greatly improves the confidence in certain variants

341 discovered by WES, particularly in highly expressed genes.

342 New somatic/GBM-related mutations evaluation from unknown variants

343 Another group of findings are shown in Table 2 as being potentially undiscovered variants, as

344 they were neither in COSMIC nor in dbSNP, but affected one of the 29 GBM-related genes and

345 retained either by SIFT or FATHMM scores. These mutations are therefore the best candidates

346 for being new discoveries as they implicate known GBM-related pathways. RNA-seq-only data

347 allowed the discovery of 8/9 potentially new mutations, against only one new variant in WES-

348 only data, which suggests that variant calling from RNA-seq has considerable potential to

349 generate new discoveries, including in already well known pathways. For example, an RNA-seq-

350 only variant, EGFR-L718R, showed 22 variant reads out of a total of 4792 (AF $4.5 \times 10^{-8}$ ). WES

351 showed 101 reads at the same position, giving a probability of only 0.39 of at least one variant 
352 read occurring in the WES data (based on binomial probability). Interestingly, COSMIC has

353 cataloged a different variant, L718M at the same position (Table 2).

354 In order to confirm the overall findings from the preceding analysis, we repeated the entire

355 pipeline on an independent validation dataset comprising 15 GBM tumors downloaded from

356 TCGA. The overall characteristics of the variants in this validation dataset, as well as the

357 genomic locations, the nature of variants found in the WES, RNA-seq-only and Intersection sets,

358 and the identities of genes showing significant RNA-seq-only variants matched well with our

359 previous analysis (Supplementary Fig. S6).

\section{DISCUSSION}

361 Although WES has been the mainstay of somatic mutation identification in cancer genomes, our

362 study suggests that variant calling from RNA-seq offers a valuable complement. RNA-seq

363 revealed new variants that were clearly associated with GBM biology, were found at the same

364 positions as previously known variants, and yet were missed by WES. A major reason for the

365 ability of RNA-seq to identify new somatic variants likely comes from the higher sequencing

366 coverage of strongly expressed genes. Oncogenes in cancers, such as EGFR in GBM, are likely

367 to be highly expressed, and RNA-seq naturally provides better coverage of such genes than

368 WES, and hence higher statistical confidence to detect variants. Additionally, even when tumor

369 cells expressing active oncogenes comprise only a subset of the tumor, RNA-seq reads can

370 capture this overrepresentation when RNA is isolated from the bulk tumor, whereas DNA used

371 for WES cannot. In this regard, RNA-seq is likely to be advantageous even over whole-genome

372 sequencing, where it is harder to achieve the same depth of coverage over all genes as WES. 
373 The RNA-seq variants we identified in our analysis did not appear to have significantly lower

374 quality than WES variants, although we saw a high number of variants with $\mathrm{AF}>0.95$ and low

375 coverage in RNA-seq data. Based on MuTect2 output, RNA-seq detected more somatic

376 mutations than WES in the TCGA samples. However, some RNA-seq variants could be

377 considered questionable, since RNA-seq data has been shown to be more prone to false positive

378 calls (Cirulli et al. 2010), in part due to errors during the RNA to cDNA conversion, mapping

379 mismatches (Bass et al., 2012) or RNA editing processes (Danecek et al. 2012). Indels are also a

380 source of possible artefacts (Kroigard et al. 2016) even though the local de novo assembly done

381 by MuTect2 should reduce this artefact. Comparison of variants with known somatic mutations

382 from the COSMIC database showed that WES-only data contained more COSMIC variants than

383 RNA-seq-only in TCGA samples (323 vs 138, Fig. 3E). However, this representation is likely to

384 be skewed by the fact that COSMIC variants were primarily discovered by WES. Variants in

385 coding regions were represented in the same proportions in RNA-seq and WES (see Fig. 3C) and

386 overrepresented in the intersection, suggesting that RNA-seq and WES coverage have a higher

387 overlap in coding regions, and making it possible to compare mutations found in both datasets

388 within coding regions. We focused on variants causing an amino-acid change, for which

389 functional impact could be estimated with the scoring systems SIFT and FATHMM. To assess

390 the identification of potential cancer-drivers that were specific to GBM, we evaluated the

391 recovery of variants in 29 genes within specific pathways previously shown to be altered in

392 GBM by a TCGA study (Cancer Genome Atlas Research Network 2008). By this measure,

393 RNA-seq-only data detected 3 out of 11 possible variants while WES-only detected 2 out of 11 ,

394 even though COSMIC variants have been primarily discovered through WES. The intersection

395 recovered 6 out of these 11 variants (see Table 1). Strikingly, RNA-seq-only data outperformed 
396 WES-only in discovering new mutations falling into these 29 GBM-related genes (8/9 findings).

397 RNA-seq-only is thus able to not only detect already known mutations, but detect potentially

398 new mutations falling into known GBM-related pathways, despite the high sequencing depth of

399 WES. In sum RNA-seq was able to find 9 of 11 key known mutations and 8 new discoveries,

400 justifying its use for variant discovery in cancer. RNA-seq data had the potential to better detect

401 variants showing very low allelic fraction (Cirulli et al. 2010), when more reads were available

402 in highly expressed genes. Analysis on the coverage indeed showed numerous variants showing

403 low AF and high coverage and therefore likely to be missed by WES alone. Additionally, a

404 previous study has shown that RNA-seq-only variants tend to be missed by WES mainly because

405 they fall outside WES capture kit boundaries ( 71\% of RNA-seq-only variants vs WES), and

406 tend to be located in highly expressed genes, which are more likely to be related to cancer than

407 unexpressed genes, the ones falling into WES-only data (Cirulli et al. 2010).

408 Several ways of improving the detection of cancer-related mutations using RNA-seq are

409 possible. First, it may be possible to optimize the pipeline by reducing artefacts and germline

410 content. A recent study developed a pipeline for analysis of variants in RNA-seq data (Piskol et

411 al. 2013). They used an indel realignment step and called variants in a more permissive way for

412 RNA-seq but at the same time requiring better base quality scores. After variant calling, they

413 filtered out known RNA editing sites using the RADAR database (Ramaswami \& Li 2014).

414 Second, a variant filtering step using a machine-learning approach could be used to train a model

415 with MuTect2 output features specifically for RNA-seq data (Spinella et al. 2016). Third, RNA-

416 seq read generators such as BEERS (Grant et al. 2011) or Flux simulator (Griebel et al. 2012)

417 could be used to optimize the pipeline by fine-tuning the sensitivity/specificity. 


\section{CONCLUSIONS}

419 Somatic mutations in tumors can be identified from RNA-seq data as a complement to exome 420 sequencing. Some mutations we identified in GBM based on RNA-seq data occurred in genes

421 known to be related to GBM and were missed by exome sequencing alone. In many cases,

422 different variants at the same positions were cataloged in the COSMIC database of somatic

423 mutations in cancer. The use of RNA-seq can thus potentially reveal new somatic mutations

424 underlying cancer. Our work suggests that since the majority of studies on cancer-driving

425 mutations used WES-only, they are likely to have missed some key driver mutations that might

426 be found using complementary RNA-seq datasets from the same tumors.

\section{ACKNOWLEDGMENTS}

428 We thank M. Shpak, M. Cowperthwaite and A. W. Hall for tumor specimens and data. We thank 429 the Next Generation Sequencing Core Facility at the University of Texas MD Anderson Cancer

430 Center Science Park for sequencing, and the Texas Advanced Computing Center (TACC) and

431 the Biomedical Research Computing Facility (BRCF) at The University of Texas at Austin for

432 HPC resources and computational support. This work is based in part based upon data generated

433 by The Cancer Genome Atlas managed by the NCI and NHGRI for which we are grateful.

434

435

436

437

438

439

440

441

442

443

444

445

\section{REFERENCES}

Alioto TS, Buchhalter I, Derdak S, Hutter B, Eldridge MD, Hovig E, Heisler LE, Beck TA, Simpson JT, Tonon L, Sertier AS, Patch AM, Jager N, Ginsbach P, Drews R, Paramasivam N, Kabbe R, Chotewutmontri S, Diessl N, Previti C, Schmidt S, Brors B, Feuerbach L, Heinold M, Grobner S, Korshunov A, Tarpey PS, Butler AP, Hinton J, Jones D, Menzies A, Raine K, Shepherd R, Stebbings L, Teague JW, Ribeca P, Giner FC, Beltran S, Raineri E, Dabad M, Heath SC, Gut M, Denroche RE, Harding NJ, Yamaguchi TN, Fujimoto A, Nakagawa H, Quesada V, Valdes-Mas R, Nakken S, Vodak D, Bower L, Lynch AG, Anderson CL, Waddell N, Pearson JV, Grimmond SM, Peto M, Spellman P, He M, Kandoth C, Lee S, Zhang J, Letourneau L, Ma S, Seth S, Torrents D, Xi L, Wheeler DA, Lopez-Otin C, Campo E, Campbell PJ, Boutros PC, Puente XS, Gerhard DS, Pfister SM, McPherson JD, Hudson TJ, Schlesner M, Lichter P, Eils R, Jones DT, 
446

447

448

449

450

451

452

453

454

455

456

457

458

459

460

461

462

463

464

465

466

467

468

469

470

471

472

473

474

475

476

477

478

479

480

481

482

483

484

485

486

487

488

489

and Gut IG. 2015. A comprehensive assessment of somatic mutation detection in cancer using whole-genome sequencing. Nat Commun 6:10001. 10.1038/ncomms10001

Berger AH, Brooks AN, Wu X, Shrestha Y, Chouinard C, Piccioni F, Bagul M, Kamburov A, Imielinski M, Hogstrom L, Zhu C, Yang X, Pantel S, Sakai R, Watson J, Kaplan N, Campbell JD, Singh S, Root DE, Narayan R, Natoli T, Lahr DL, Tirosh I, Tamayo P, Getz G, Wong B, Doench J, Subramanian A, Golub TR, Meyerson M, and Boehm JS. 2016. High-throughput Phenotyping of Lung Cancer Somatic Mutations. Cancer Cell 30:214-228. 10.1016/j.ccell.2016.06.022

Brennan CW, Verhaak RG, McKenna A, Campos B, Noushmehr H, Salama SR, Zheng S, Chakravarty D, Sanborn JZ, Berman SH, Beroukhim R, Bernard B, Wu CJ, Genovese G, Shmulevich I, Barnholtz-Sloan J, Zou L, Vegesna R, Shukla SA, Ciriello G, Yung WK, Zhang W, Sougnez C, Mikkelsen T, Aldape K, Bigner DD, Van Meir EG, Prados M, Sloan A, Black KL, Eschbacher J, Finocchiaro G, Friedman W, Andrews DW, Guha A, Iacocca M, O'Neill BP, Foltz G, Myers J, Weisenberger DJ, Penny R, Kucherlapati R, Perou CM, Hayes DN, Gibbs R, Marra M, Mills GB, Lander E, Spellman P, Wilson R, Sander C, Weinstein J, Meyerson M, Gabriel S, Laird PW, Haussler D, Getz G, Chin L, and Network TR. 2013. The somatic genomic landscape of glioblastoma. Cell 155:462477. 10.1016/j.cell.2013.09.034

Campbell CD, and Eichler EE. 2013. Properties and rates of germline mutations in humans. Trends Genet 29:575-584. 10.1016/j.tig.2013.04.005

Cancer Genome Atlas Research Network. 2008. Comprehensive genomic characterization defines human glioblastoma genes and core pathways. Nature 455:1061-1068. 10.1038/nature07385

Cancer Genome Atlas Research Network. 2013. The Cancer Genome Atlas Pan-Cancer analysis project. Nat Genet 45:1113-1120. 10.1038/ng.2764

Cavalcante Mde S, Torres-Romero JC, Lobo MD, Moreno FB, Bezerra LP, Lima DS, Matos JC, Moreira Rde A, and Monteiro-Moreira AC. 2016. A panel of glycoproteins as candidate biomarkers for early diagnosis and treatment evaluation of B-cell acute lymphoblastic leukemia. Biomark Res 4:1. 10.1186/s40364-016-0055-6

Cibulskis K, Lawrence MS, Carter SL, Sivachenko A, Jaffe D, Sougnez C, Gabriel S, Meyerson M, Lander ES, and Getz G. 2013. Sensitive detection of somatic point mutations in impure and heterogeneous cancer samples. Nat Biotechnol 31:213-219. 10.1038/nbt.2514

Cirulli ET, Singh A, Shianna KV, Ge D, Smith JP, Maia JM, Heinzen EL, Goedert JJ, Goldstein DB, and Center for HIVAVI. 2010. Screening the human exome: a comparison of whole genome and whole transcriptome sequencing. Genome Biol 11:R57. 10.1186/gb-201011-5-r57

Danecek P, Nellaker C, McIntyre RE, Buendia-Buendia JE, Bumpstead S, Ponting CP, Flint J, Durbin R, Keane TM, and Adams DJ. 2012. High levels of RNA-editing site conservation amongst 15 laboratory mouse strains. Genome Biol 13:26. 10.1186/gb2012-13-4-r26

Ding J, Bashashati A, Roth A, Oloumi A, Tse K, Zeng T, Haffari G, Hirst M, Marra MA, Condon A, Aparicio S, and Shah SP. 2012. Feature-based classifiers for somatic mutation detection in tumour-normal paired sequencing data. Bioinformatics 28:167-175. 10.1093/bioinformatics/btr629 
490

491

492

493

494

495

496

497

498

499

500

501

502

503

504

505

506

507

508

509

510

511

512

513

514

515

516

517

518

519

520

521

522

523

524

525

526

527

528

529

530

531

532

533

534

Dobin A, Davis CA, Schlesinger F, Drenkow J, Zaleski C, Jha S, Batut P, Chaisson M, and Gingeras TR. 2013. STAR: ultrafast universal RNA-seq aligner. Bioinformatics 29:1521. 10.1093/bioinformatics/bts635

Dobin A, and Gingeras TR. 2015. Mapping RNA-seq Reads with STAR. Curr Protoc Bioinformatics 51:11 14 11-19. 10.1002/0471250953.bi1114s51

Forbes SA, Beare D, Gunasekaran P, Leung K, Bindal N, Boutselakis H, Ding M, Bamford S, Cole C, Ward S, Kok CY, Jia M, De T, Teague JW, Stratton MR, McDermott U, and Campbell PJ. 2015. COSMIC: exploring the world's knowledge of somatic mutations in human cancer. Nucleic Acids Res 43:D805-811. 10.1093/nar/gku1075

Global Burden of Disease Cancer Collaboration. 2017. Global, Regional, and National Cancer Incidence, Mortality, Years of Life Lost, Years Lived With Disability, and DisabilityAdjusted Life-years for 32 Cancer Groups, 1990 to 2015: A Systematic Analysis for the Global Burden of Disease Study. JAMA Oncol 3:524-548. 10.1001/jamaoncol.2016.5688

Grant GR, Farkas MH, Pizarro AD, Lahens NF, Schug J, Brunk BP, Stoeckert CJ, Hogenesch JB, and Pierce EA. 2011. Comparative analysis of RNA-Seq alignment algorithms and the RNA-Seq unified mapper (RUM). Bioinformatics 27:2518-2528. 10.1093/bioinformatics/btr427

Griebel T, Zacher B, Ribeca P, Raineri E, Lacroix V, Guigo R, and Sammeth M. 2012. Modelling and simulating generic RNA-Seq experiments with the flux simulator. Nucleic Acids Res 40:10073-10083. 10.1093/nar/gks666

Hall AW, Battenhouse AM, Shivram H, Morris AR, Cowperthwaite MC, Shpak M, and Iyer VR. 2018. Bivalent Chromatin Domains in Glioblastoma Reveal a Subtype-Specific Signature of Glioma Stem Cells. Cancer Res 78:2463-2474. 10.1158/0008-5472.CAN-17-1724

Hanahan D, and Weinberg RA. 2011. Hallmarks of cancer: the next generation. Cell 144:646674. 10.1016/j.cell.2011.02.013

Harrow J, Frankish A, Gonzalez JM, Tapanari E, Diekhans M, Kokocinski F, Aken BL, Barrell D, Zadissa A, Searle S, Barnes I, Bignell A, Boychenko V, Hunt T, Kay M, Mukherjee G, Rajan J, Despacio-Reyes G, Saunders G, Steward C, Harte R, Lin M, Howald C, Tanzer A, Derrien T, Chrast J, Walters N, Balasubramanian S, Pei B, Tress M, Rodriguez JM, Ezkurdia I, van Baren J, Brent M, Haussler D, Kellis M, Valencia A, Reymond A, Gerstein M, Guigo R, and Hubbard TJ. 2012. GENCODE: the reference human genome annotation for The ENCODE Project. Genome Res 22:1760-1774.

$10.1101 /$ gr. 135350.111

$\mathrm{Hu}$, and Ng PC. 2013. SIFT Indel: predictions for the functional effects of amino acid insertions/deletions in proteins. PLoS One 8:e77940. 10.1371/journal.pone.0077940

Kiran AM, O'Mahony JJ, Sanjeev K, and Baranov PV. 2013. Darned in 2013: inclusion of model organisms and linking with Wikipedia. Nucleic Acids Res 41:D258-261. 10.1093/nar/gks961

Kitts A, Phan L, Ward M, and Holmes JB. 2013. The Database of Short Genetic Variation (dbSNP). National Center for Biotechnology Information (US).

Kroigard AB, Thomassen M, Laenkholm AV, Kruse TA, and Larsen MJ. 2016. Evaluation of Nine Somatic Variant Callers for Detection of Somatic Mutations in Exome and Targeted Deep Sequencing Data. PLoS One 11:e0151664. 10.1371/journal.pone.0151664

Li H, and Durbin R. 2009. Fast and accurate short read alignment with Burrows-Wheeler transform. Bioinformatics 25:1754-1760. 10.1093/bioinformatics/btp324 
535

536

537

538

539

540

541

542

543

544

545

546

547

548

549

550

551

552

553

554

555

556

557

558

559

560

561

562

563

564

565

566

567

568

569

570

571

572

573

574

575

576

577

578

579

580

Martin M. 2011. Cutadapt removes adapter sequences from high-throughput sequencing reads. EMBnetjournal 17:10-12. 10.14806/ej.17.1.200

Meacham CE, and Morrison SJ. 2013. Tumour heterogeneity and cancer cell plasticity. Nature 501:328-337. 10.1038/nature12624

Mouri A, Noda Y, Watanabe K, and Nabeshima T. 2013. The roles of MAGE-D1 in the neuronal functions and pathology of the central nervous system. Rev Neurosci 24:61-70. 10.1515/revneuro-2012-0069

Nadarajah N, Meggendorfer M, Kern W, Haferlach C, and Haferlach T. 2016. Significance assessment of mutations in 944 MDS patients using publicly available variant databases and mutation impact prediction software. [abstract]. In: Proceedings of the 107th Annual Meeting of the American Association for Cancer Research; 2016 Apr 16-20; New Orleans, LA. Cancer Research 76 (14 suppl):1514. https://doi.org/10.1158/15387445.AM2016-1514

Neuzillet C, Tijeras-Raballand A, Cros J, Faivre S, Hammel P, and Raymond E. 2013. Stromal expression of SPARC in pancreatic adenocarcinoma. Cancer Metastasis Rev 32:585-602. 10.1007/s10555-013-9439-3

Ng PC, and Henikoff S. 2003. SIFT: Predicting amino acid changes that affect protein function. Nucleic Acids Res 31:3812-3814.

O'Brien TD, Jia P, Xia J, Saxena U, Jin H, Vuong H, Kim P, Wang Q, Aryee MJ, MinoKenudson M, Engelman JA, Le LP, Iafrate AJ, Heist RS, Pao W, and Zhao Z. 2015. Inconsistency and features of single nucleotide variants detected in whole exome sequencing versus transcriptome sequencing: A case study in lung cancer. Methods 83:118-127. 10.1016/j.ymeth.2015.04.016

O'Leary NA, Wright MW, Brister JR, Ciufo S, Haddad D, McVeigh R, Rajput B, Robbertse B, Smith-White B, Ako-Adjei D, Astashyn A, Badretdin A, Bao Y, Blinkova O, Brover V, Chetvernin V, Choi J, Cox E, Ermolaeva O, Farrell CM, Goldfarb T, Gupta T, Haft D, Hatcher E, Hlavina W, Joardar VS, Kodali VK, Li W, Maglott D, Masterson P, McGarvey KM, Murphy MR, O'Neill K, Pujar S, Rangwala SH, Rausch D, Riddick LD, Schoch C, Shkeda A, Storz SS, Sun H, Thibaud-Nissen F, Tolstoy I, Tully RE, Vatsan AR, Wallin C, Webb D, Wu W, Landrum MJ, Kimchi A, Tatusova T, DiCuccio M, Kitts P, Murphy TD, and Pruitt KD. 2016. Reference sequence (RefSeq) database at NCBI: current status, taxonomic expansion, and functional annotation. Nucleic Acids Res 44:D733-745. 10.1093/nar/gkv1189

Paez JG, Janne PA, Lee JC, Tracy S, Greulich H, Gabriel S, Herman P, Kaye FJ, Lindeman N, Boggon TJ, Naoki K, Sasaki H, Fujii Y, Eck MJ, Sellers WR, Johnson BE, and Meyerson M. 2004. EGFR mutations in lung cancer: correlation with clinical response to gefitinib therapy. Science 304:1497-1500. 10.1126/science.1099314

Picardi E, Manzari C, Mastropasqua F, Aiello I, D'Erchia AM, and Pesole G. 2015. Profiling RNA editing in human tissues: towards the inosinome Atlas. Sci Rep 5:14941. 10.1038/srep14941

Pickrell JK, Pai AA, Gilad Y, and Pritchard JK. 2010. Noisy splicing drives mRNA isoform diversity in human cells. PLoS Genet 6:e1001236. 10.1371/journal.pgen.1001236

Piskol R, Ramaswami G, and Li JB. 2013. Reliable identification of genomic variants from RNA-seq data. Am J Hum Genet 93:641-651. 10.1016/j.ajhg.2013.08.008

Puente XS, Pinyol M, Quesada V, Conde L, Ordonez GR, Villamor N, Escaramis G, Jares P, Bea S, Gonzalez-Diaz M, Bassaganyas L, Baumann T, Juan M, Lopez-Guerra M, Colomer D, 
581

582

583

584

585

586

587

588

589

590

591

592

593

594

595

596

597

598

599

600

601

602

603

604

605

606

607

608

609

610

611

612

613

614

615

616

617

618

619

620

621

622

623

624

625

Tubio JM, Lopez C, Navarro A, Tornador C, Aymerich M, Rozman M, Hernandez JM, Puente DA, Freije JM, Velasco G, Gutierrez-Fernandez A, Costa D, Carrio A, Guijarro S, Enjuanes A, Hernandez L, Yague J, Nicolas P, Romeo-Casabona CM, Himmelbauer H, Castillo E, Dohm JC, de Sanjose S, Piris MA, de Alava E, San Miguel J, Royo R, Gelpi JL, Torrents D, Orozco M, Pisano DG, Valencia A, Guigo R, Bayes M, Heath S, Gut M, Klatt P, Marshall J, Raine K, Stebbings LA, Futreal PA, Stratton MR, Campbell PJ, Gut I, Lopez-Guillermo A, Estivill X, Montserrat E, Lopez-Otin C, and Campo E. 2011. Whole-genome sequencing identifies recurrent mutations in chronic lymphocytic leukaemia. Nature 475:101-105. 10.1038/nature10113

Ramaswami G, and Li JB. 2014. RADAR: a rigorously annotated database of A-to-I RNA editing. Nucleic Acids Res 42:D109-113. 10.1093/nar/gkt996

Raphael BJ, Dobson JR, Oesper L, and Vandin F. 2014. Identifying driver mutations in sequenced cancer genomes: computational approaches to enable precision medicine. Genome Med 6:5. 10.1186/gm524

Reis ES, Mastellos DC, Ricklin D, Mantovani A, and Lambris JD. 2018. Complement in cancer: untangling an intricate relationship. Nat Rev Immunol 18:5-18. 10.1038/nri.2017.97

Rhee JK, Lee S, Park WY, Kim YH, and Kim TM. 2017. Allelic imbalance of somatic mutations in cancer genomes and transcriptomes. Sci Rep 7:1653. 10.1038/s41598-017-01966-Z

Sahni N, Yi S, Zhong Q, Jailkhani N, Charloteaux B, Cusick ME, and Vidal M. 2013. Edgotype: a fundamental link between genotype and phenotype. Curr Opin Genet Dev 23:649-657. 10.1016/j.gde.2013.11.002

Saratsis AM, Kambhampati M, Snyder K, Yadavilli S, Devaney JM, Harmon B, Hall J, Raabe EH, An P, Weingart M, Rood BR, Magge SN, MacDonald TJ, Packer RJ, and Nazarian J. 2014. Comparative multidimensional molecular analyses of pediatric diffuse intrinsic pontine glioma reveals distinct molecular subtypes. Acta Neuropathol 127:881-895. 10.1007/s00401-013-1218-2

Shihab HA, Gough J, Cooper DN, Day IN, and Gaunt TR. 2013a. Predicting the functional consequences of cancer-associated amino acid substitutions. Bioinformatics 29:15041510. 10.1093/bioinformatics/btt182

Shihab HA, Gough J, Cooper DN, Stenson PD, Barker GL, Edwards KJ, Day IN, and Gaunt TR. $2013 \mathrm{~b}$. Predicting the functional, molecular, and phenotypic consequences of amino acid substitutions using hidden Markov models. Hum Mutat 34:57-65. 10.1002/humu.22225

Shihab HA, Rogers MF, Gough J, Mort M, Cooper DN, Day IN, Gaunt TR, and Campbell C. 2015. An integrative approach to predicting the functional effects of non-coding and coding sequence variation. Bioinformatics 31:1536-1543. 10.1093/bioinformatics/btv009

Shinoura N, Heffelfinger SC, Miller M, Shamraj OI, Miura NH, Larson JJ, DeTribolet N, Warnick RE, Tew JJ, and Menon AG. 1994. RNA expression of complement regulatory proteins in human brain tumors. Cancer Lett 86:143-149.

Sowalsky AG, Xia Z, Wang L, Zhao H, Chen S, Bubley GJ, Balk SP, and Li W. 2015. Whole transcriptome sequencing reveals extensive unspliced mRNA in metastatic castrationresistant prostate cancer. Mol Cancer Res 13:98-106. 10.1158/1541-7786.MCR-14-0273

Spinella JF, Mehanna P, Vidal R, Saillour V, Cassart P, Richer C, Ouimet M, Healy J, and Sinnett D. 2016. SNooPer: a machine learning-based method for somatic variant identification from low-pass next-generation sequencing. BMC Genomics 17:912. $10.1186 / \mathrm{s} 12864-016-3281-2$ 
626

627

628

629

630

631

632

633

634

635

636

637

638

639

640

641

642

643

644

645

646

647

648

649

650

651

652

653

654

655

656

657

658

659

660

661

662

663

664

665

666

667

668

669

Stavrovskaya AA, Shushanov SS, and Rybalkina EY. 2016. Problems of Glioblastoma Multiforme Drug Resistance. Biochemistry (Mosc) 81:91-100. 10.1134/S0006297916020036

Stupp R, Hegi ME, Mason WP, van den Bent MJ, Taphoorn MJ, Janzer RC, Ludwin SK, Allgeier A, Fisher B, Belanger K, Hau P, Brandes AA, Gijtenbeek J, Marosi C, Vecht CJ, Mokhtari K, Wesseling P, Villa S, Eisenhauer E, Gorlia T, Weller M, Lacombe D, Cairncross JG, Mirimanoff RO, European Organisation for R, Treatment of Cancer Brain T, Radiation Oncology G, and National Cancer Institute of Canada Clinical Trials G. 2009. Effects of radiotherapy with concomitant and adjuvant temozolomide versus radiotherapy alone on survival in glioblastoma in a randomised phase III study: 5-year analysis of the EORTC-NCIC trial. Lancet Oncol 10:459-466. 10.1016/S14702045(09)70025-7

Suman S, Basak T, Gupta P, Mishra S, Kumar V, Sengupta S, and Shukla Y. 2016. Quantitative proteomics revealed novel proteins associated with molecular subtypes of breast cancer. $J$ Proteomics 148:183-193. 10.1016/j.jprot.2016.07.033

Takiar V, Ip CK, Gao M, Mills GB, and Cheung LW. 2017. Neomorphic mutations create therapeutic challenges in cancer. Oncogene 36:1607-1618. 10.1038/onc.2016.312

Taylor TE, Furnari FB, and Cavenee WK. 2012. Targeting EGFR for treatment of glioblastoma: molecular basis to overcome resistance. Curr Cancer Drug Targets 12:197-209.

Van der Auwera GA. 2014. Calling variants in RNAseq. Available at https://gatkforums.broadinstitute.org/gatk/discussion/3891/calling-variants-in-RNA-seq (accessed Dec 22 2017).

Van der Auwera GA, Carneiro MO, Hartl C, Poplin R, Del Angel G, Levy-Moonshine A, Jordan T, Shakir K, Roazen D, Thibault J, Banks E, Garimella KV, Altshuler D, Gabriel S, and DePristo MA. 2013. From FastQ data to high confidence variant calls: the Genome Analysis Toolkit best practices pipeline. Curr Protoc Bioinformatics 43:11 10 11-33. 10.1002/0471250953.bi1110s43

Wang K, Li M, and Hakonarson H. 2010. ANNOVAR: functional annotation of genetic variants from high-throughput sequencing data. Nucleic Acids Res 38:e164. 10.1093/nar/gkq603

Xu Y, Bismar TA, Su J, Xu B, Kristiansen G, Varga Z, Teng L, Ingber DE, Mammoto A, Kumar R, and Alaoui-Jamali MA. 2010. Filamin A regulates focal adhesion disassembly and suppresses breast cancer cell migration and invasion. $J$ Exp Med 207:2421-2437. 10.1084/jem.20100433

Yin D, Ogawa S, Kawamata N, Tunici P, Finocchiaro G, Eoli M, Ruckert C, Huynh T, Liu G, Kato M, Sanada M, Jauch A, Dugas M, Black KL, and Koeffler HP. 2009. Highresolution genomic copy number profiling of glioblastoma multiforme by single nucleotide polymorphism DNA microarray. Mol Cancer Res 7:665-677. 10.1158/15417786.MCR-08-0270

Yu B, O'Toole SA, and Trent RJ. 2015. Somatic DNA mutation analysis in targeted therapy of solid tumours. Transl Pediatr 4:125-138. 10.3978/j.issn.2224-4336.2015.04.04

\section{FIGURE LEGENDS}

Figure 1. Pipeline used to detect RNA-seq variants.

(A) Principal steps in the pipeline used to identify and annotate somatic mutations. Mutation calling was done for each paired tumor sample/matched-normal. An RNA-seq-specific panel-of- 
670 normals (PoN) and a WES-specific PoN were generated. (B) Distinction between pipelines and

671 their associated methodologies for SD01 and TCGA samples, and the difference between RNA-

672 seq and WES pipeline used in this study.

673 Figure 2. Read count, filtering by MuTect2, mutation spectrum and total variant count in 674 GBM samples.

675 (A) Proportion of reads (using samtools on BAM files) before variant calling. (B) Mutation 676 spectrum indicating the type of base substitution in total RNA-seq and WES data. The Y-axis 677 shows the proportion of mutations. (C) MuTect2 filtering statistics. Proportion of variants failing 678 each MuTect2 filter. PASS stands for the proportion of variants accepted as true and somatic by 679 MuTect2. The other filters are described in Materials and Methods. (D) Total number of variants 680 for TCGA samples (averaged over 9 samples - TCGAav). ALL is the number of variants before 681 MuTect2 filtering. PASS are the ones accepted as true and somatic by MuTect2. The number 682 scale on top refers to the top two classes of variants (ALL and PASS). Coding refers to variants 683 from coding regions. Del stands for variants in coding regions inducing an AA change (non684 synonymous SNVs, frameshift indels or stop gain/loss). The number scale on bottom refers to 685 the bottom two classes of variants (Coding and Del). The actual number of variants in PASS 686 TCGAav from RNA-seq (blue), intersection (red) and WES (beige) is also indicated.

687 Figure 3. Variant features including allele fraction, coverage, genomic location and 688 COSMIC/dbSNP content in TCGA samples.

689 (A) Scatter plot representing the fraction of the altered allele estimated from altered read fraction 690 (allele fraction) versus coverage at the variant position (total number of reads). The indicated 691 intervals show the proportion of RNA-seq only variants having $\mathrm{AF}<0.05, \mathrm{AF}>0.50$ or $\mathrm{AF}>$ 692 0.95. Merged data from 9 TCGA samples is shown. (B) Histograms of the distribution of allele 
693 fraction (AF) for the indicated classes of variants. Merged data from 9 TCGA samples is shown.

694 (C) Genomic location of PASS variants, given as the average value over 9 TCGA samples. (D)

695 Type of variants from coding regions, given as the average obtained over 9 TCGA samples.

696 Indel stands for insertions and deletions. (E) Proportion of variants from coding regions included

697 in COSMIC and/or dbSNP, given as the average obtained over 9 TCGA samples. Absolute

698 numbers of COSMIC-only variants are indicated.

699 Figure 4. Heatmap of the 63 most widely mutated genes across TCGA samples in RNA-seq-

700 only.

701 Only genes altered in coding regions in at least 5 out of 9 tumor samples are shown. Rows

702 indicate genes and columns are tumors. Black indicates no variants, bright yellow only one

703 variant/gene and red 7 variants/gene, with a gradient from yellow to red indicating a number of

704 variants/gene included between 1 and 7. The heatmap was clustered by rows and columns (the

705 dendrogram is not shown).

706 Figure 5. SIFT and FATHMM scores for TCGA samples.

707 (A) Scatterplots of FATHMM scores versus SIFT scores for non-synonymous SNVs. The

708 threshold for deleterious variants for SIFT is 0.05 and the threshold for cancer-drivers for

709 FATHMM is -0.75 , which are indicated. Mutations retained by both are thus in the bottom left

710 corner of each plot. Pearson correlations between SIFT and FATHMM are indicated by R values

711 for each plot. (B) Box plots showing the distribution of FATHMM scores for each of the

712 indicated categories of variants. The only significant difference was between the Intersection and

713 WES-only groups $(p=0.0085)$. Genes with the smallest variant scores are indicated. (C) Box

714 plots showing the distribution of SIFT scores. No significant differences were observed. (D)

715 Proportion of variants retained as deleterious or cancer-driver by SIFT and/or FATHMM 
716 respectively. The proportion retained by FATHMM is indicated in red, and the proportion

717 retained by both SIFT and FATHMM is indicated in blue. 
Figure 1 (on next page)

Pipeline used to detect RNA-seq variants.

(A) Principal steps in the pipeline used to identify and annotate somatic mutations. Mutation calling was done for each paired tumor sample/matched-normal. An RNA-seq-specific panelof-normals (PoN) and a WES-specific PoN were generated. (B) Distinction between pipelines and their associated methodologies for SD01 and TCGA samples, and the difference between RNA-seq and WES pipeline used in this study. 
Tumor Blood-derived Sample 1 Matched-Normal \begin{tabular}{|l|l|l|||||||||}
\hline \multicolumn{3}{|c|}{ adapters, removing r/tRNA contaminants } \\
\hline
\end{tabular}

Alignment STAR 2-pass, bwa

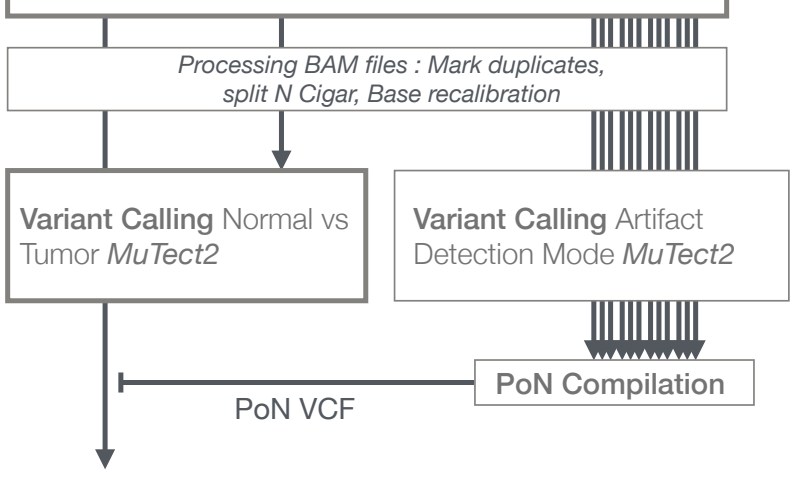

Variant list in VCF format

Other Normal

Samples

||||||||||||

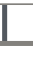




\section{Figure 2 (on next page)}

Read count, filtering by MuTect2, mutation spectrum and total variant count in GBM samples.

(A) Proportion of reads (using samtools on BAM files) before variant calling. (B) Mutation spectrum indicating the type of base substitution in total RNA-seq and WES data. The Y-axis shows the proportion of mutations. (C) MuTect2 filtering statistics. Proportion of variants failing each MuTect2 filter. PASS stands for the proportion of variants accepted as true and somatic by MuTect2. The other filters are described in Materials and Methods. (D) Total number of variants for TCGA samples (averaged over 9 samples - TCGAav). ALL is the number of variants before MuTect2 filtering. PASS are the ones accepted as true and somatic by MuTect2. The number scale on top refers to the top two classes of variants (ALL and PASS). Coding refers to variants from coding regions. Del stands for variants in coding regions inducing an AA change (non-synonymous SNVs, frameshift indels or stop gain/loss). The number scale on bottom refers to the bottom two classes of variants (Coding and Del). The actual number of variants in PASS TCGAav from RNA-seq (blue), intersection (red) and WES (beige) is also indicated. 


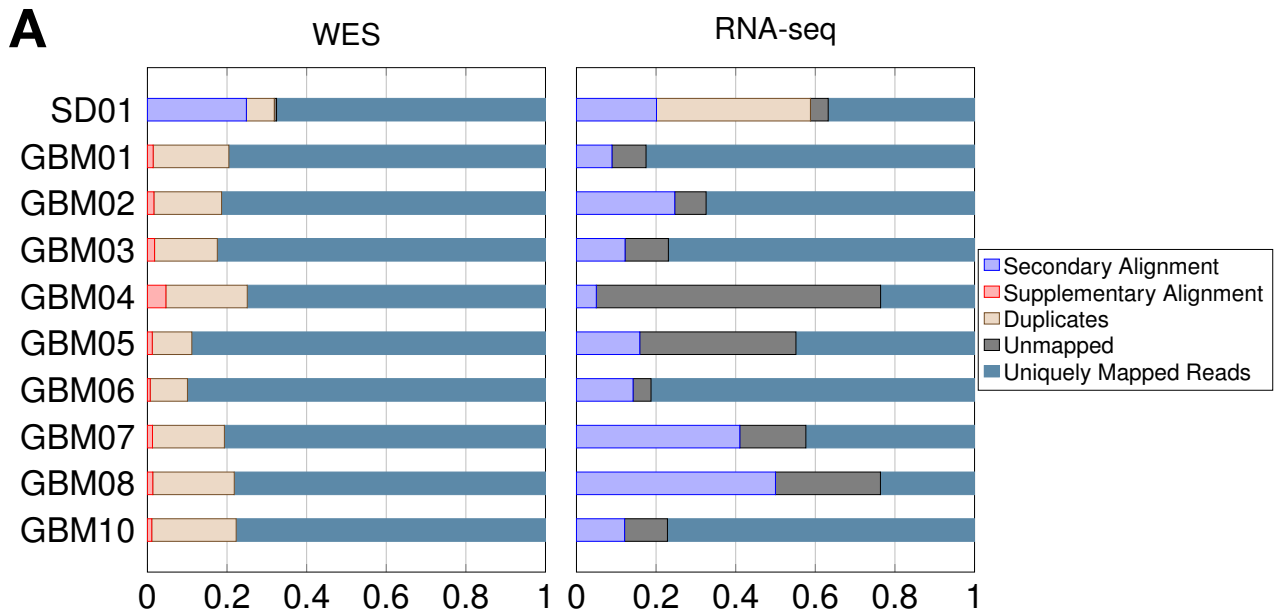

C

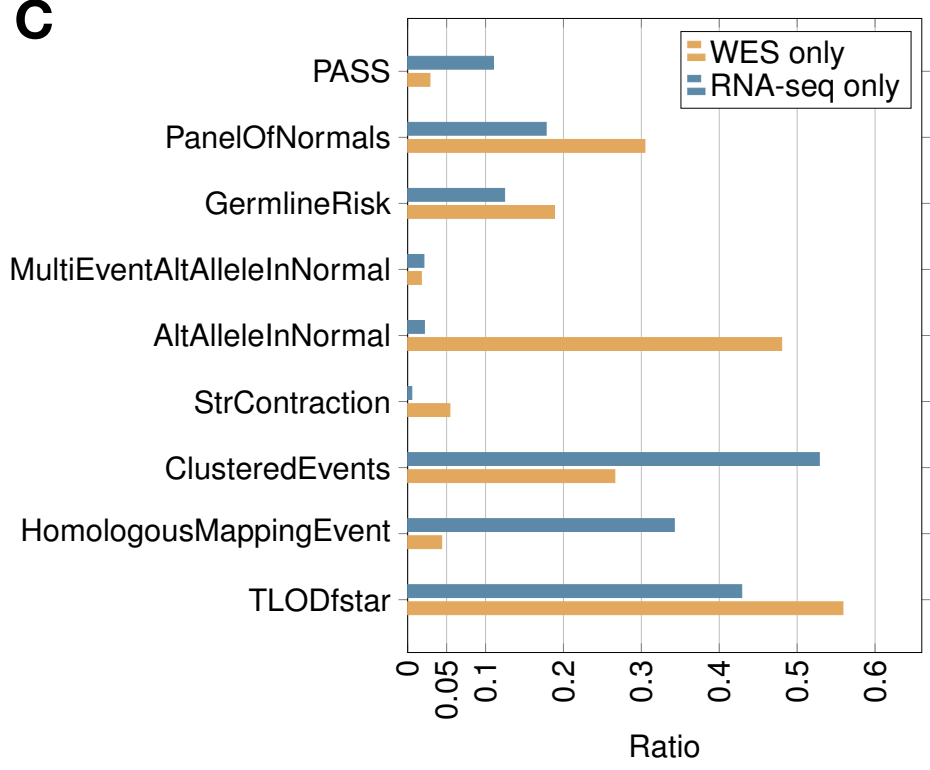

B

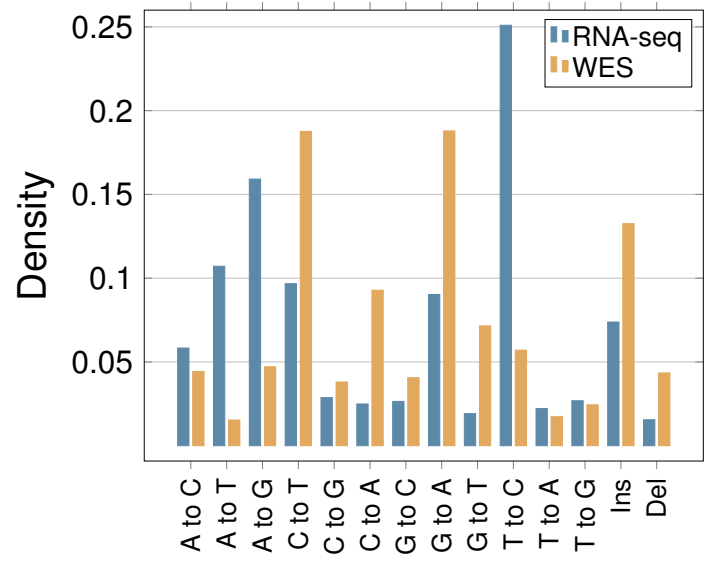

D Number of variants

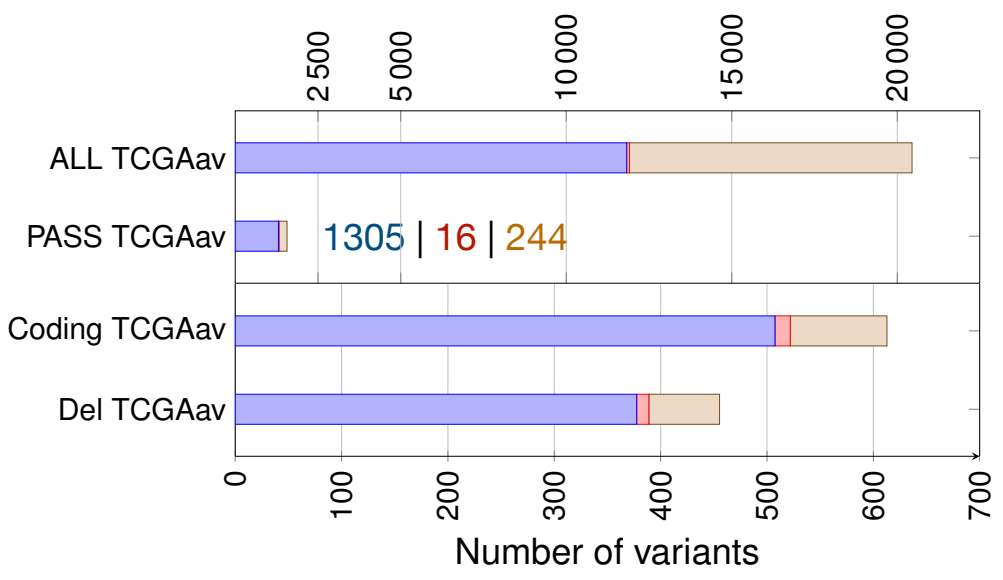

$\square$ RNA-seq only $\square$ Intersection $\square$ WES only 


\section{Figure 3 (on next page)}

Variant features including allele fraction, coverage, genomic location and COSMIC/dbSNP content in TCGA samples.

(A) Scatter plot representing the fraction of the altered allele estimated from altered read fraction (allele fraction) versus coverage at the variant position (total number of reads). The indicated intervals show the proportion of RNA-seq only variants having AF $<0.05, A F>0.50$ or AF > 0.95. Merged data from 9 TCGA samples is shown. (B) Histograms of the distribution of allele fraction (AF) for the indicated classes of variants. Merged data from 9 TCGA samples is shown. (C) Genomic location of PASS variants, given as the average value over 9 TCGA samples. (D) Type of variants from coding regions, given as the average obtained over 9 TCGA samples. Indel stands for insertions and deletions. (E) Proportion of variants from coding regions included in COSMIC and/or dbSNP, given as the average obtained over 9 TCGA samples. Absolute numbers of COSMIC-only variants are indicated. 
A

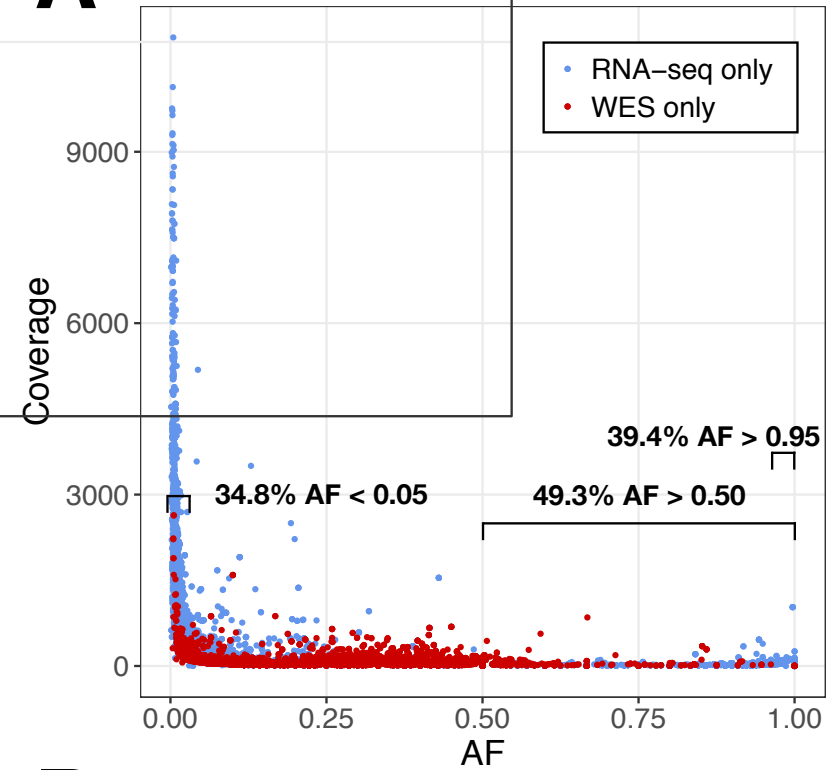

B

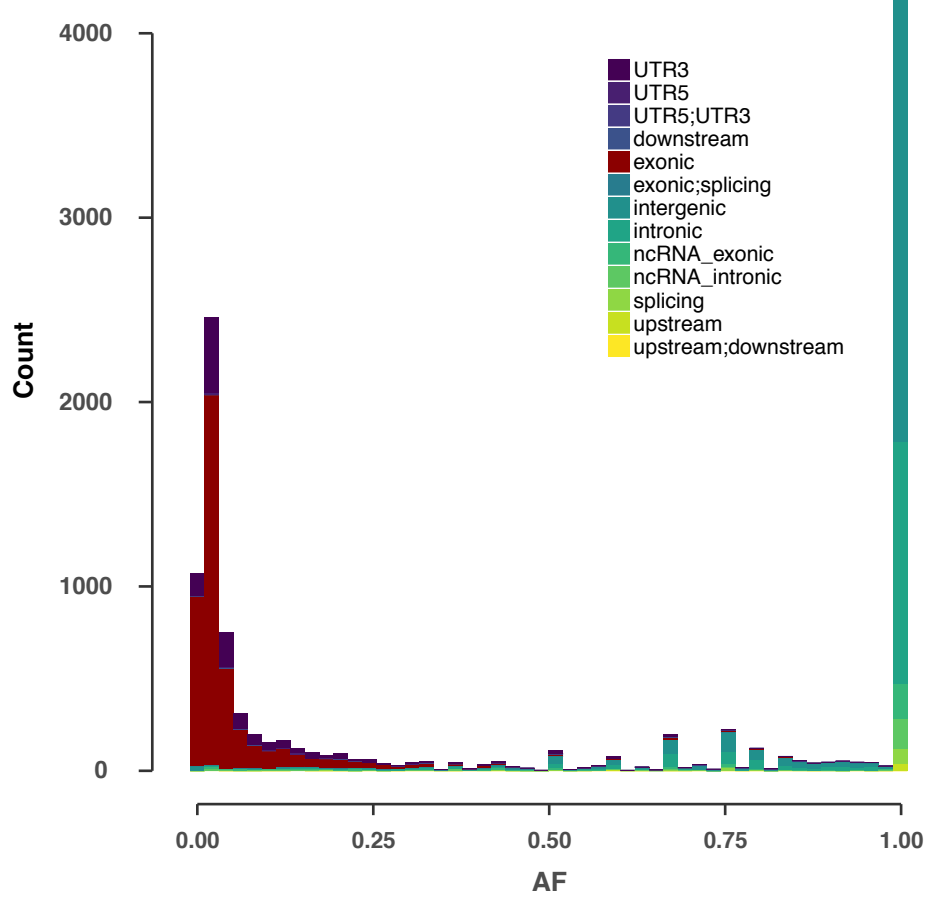

RNA-seq only

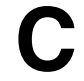

D
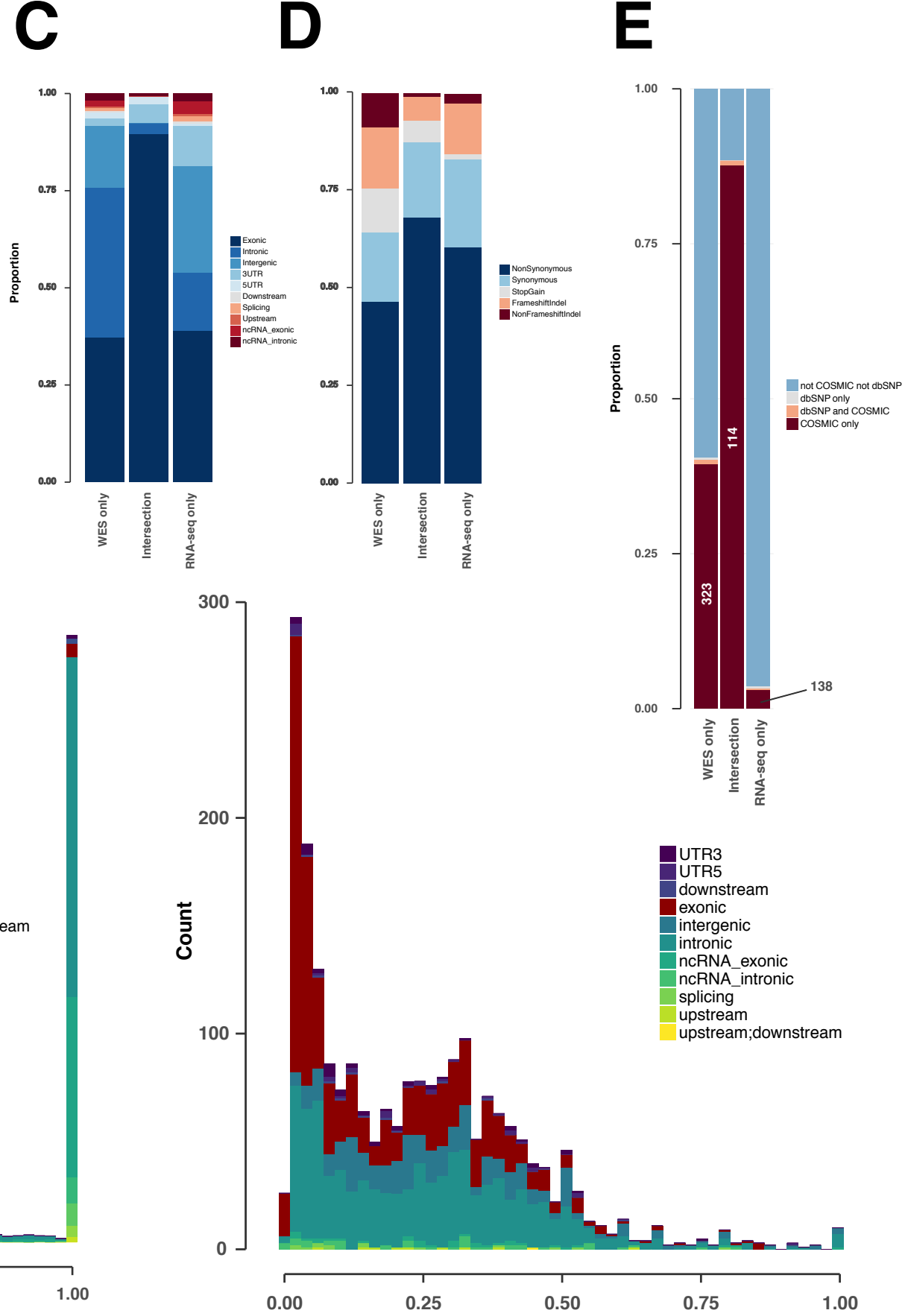

UTR5

downstream

exonic

intergenic

ncRNA exonic

ncRNA intronic

splicing

upstream

upstream;downstream

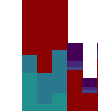

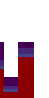
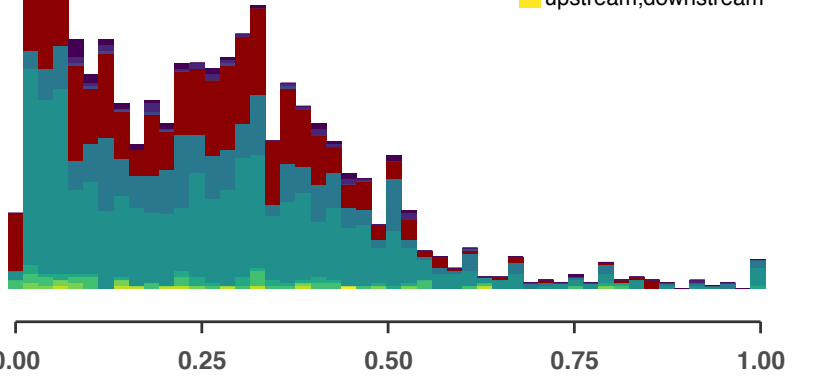

AF

WES only 
Figure 4 (on next page)

Heatmap of the 63 most widely mutated genes across TCGA samples in RNA-seq-only.

Only genes altered in coding regions in at least 5 out of 9 tumor samples are shown. Rows indicate genes and columns are tumors. Black indicates no variants, bright yellow only one variant/gene and red 7 variants/gene, with a gradient from yellow to red indicating a number of variants/gene included between 1 and 7. The heatmap was clustered by rows and columns (the dendrogram is not shown). 


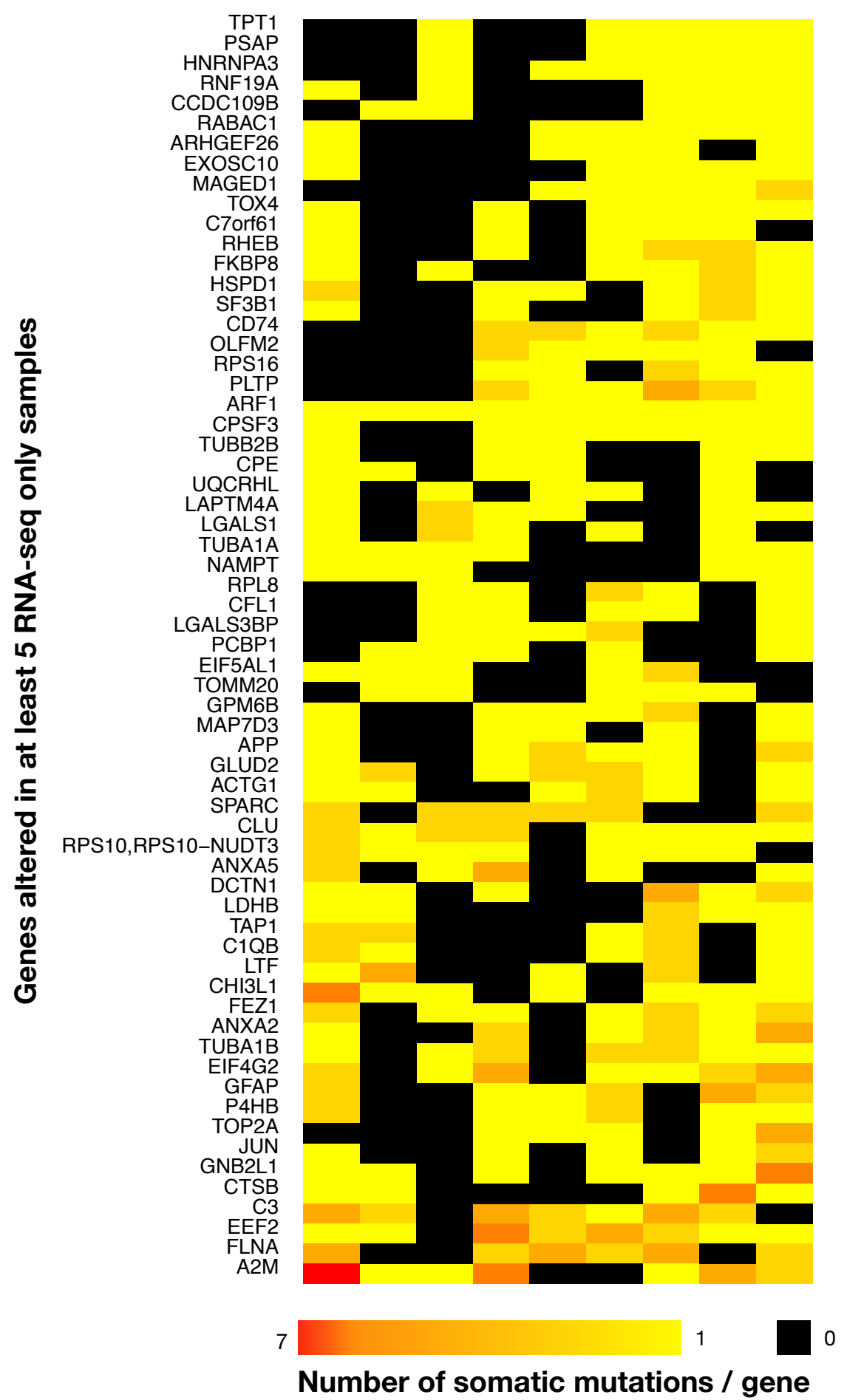




\section{Figure 5 (on next page)}

SIFT and FATHMM scores for TCGA samples.

(A) Scatterplots of FATHMM scores versus SIFT scores for non-synonymous SNVs. The threshold for deleterious variants for SIFT is 0.05 and the threshold for cancer-drivers for FATHMM is -0.75 , which are indicated. Mutations retained by both are thus in the bottom left corner of each plot. Pearson correlations between SIFT and FATHMM are indicated by $\mathrm{R}$ values for each plot. (B) Box plots showing the distribution of FATHMM scores for each of the indicated categories of variants. The only significant difference was between the Intersection and WES-only groups ( $p=0.0085)$. Genes with the smallest variant scores are indicated. (C) Box plots showing the distribution of SIFT scores. No significant differences were observed. (D) Proportion of variants retained as deleterious or cancer-driver by SIFT and/or FATHMM respectively. The proportion retained by FATHMM is indicated in red, and the proportion retained by both SIFT and FATHMM is indicated in blue. 
A

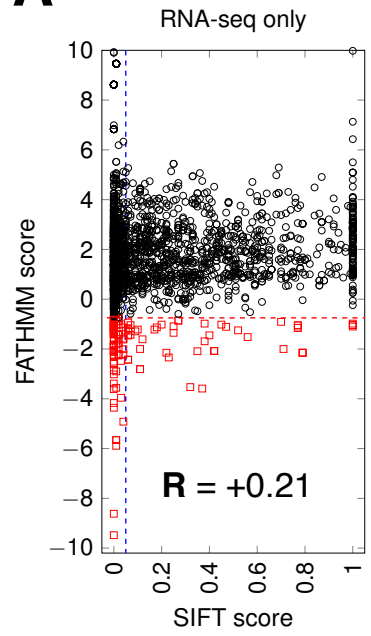

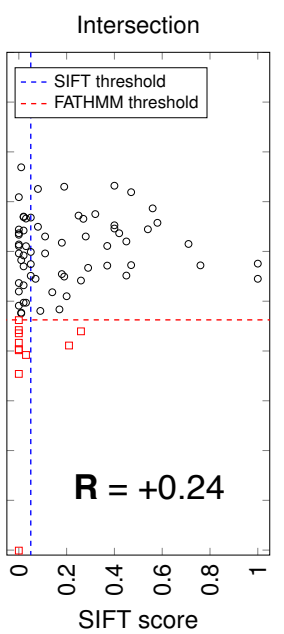
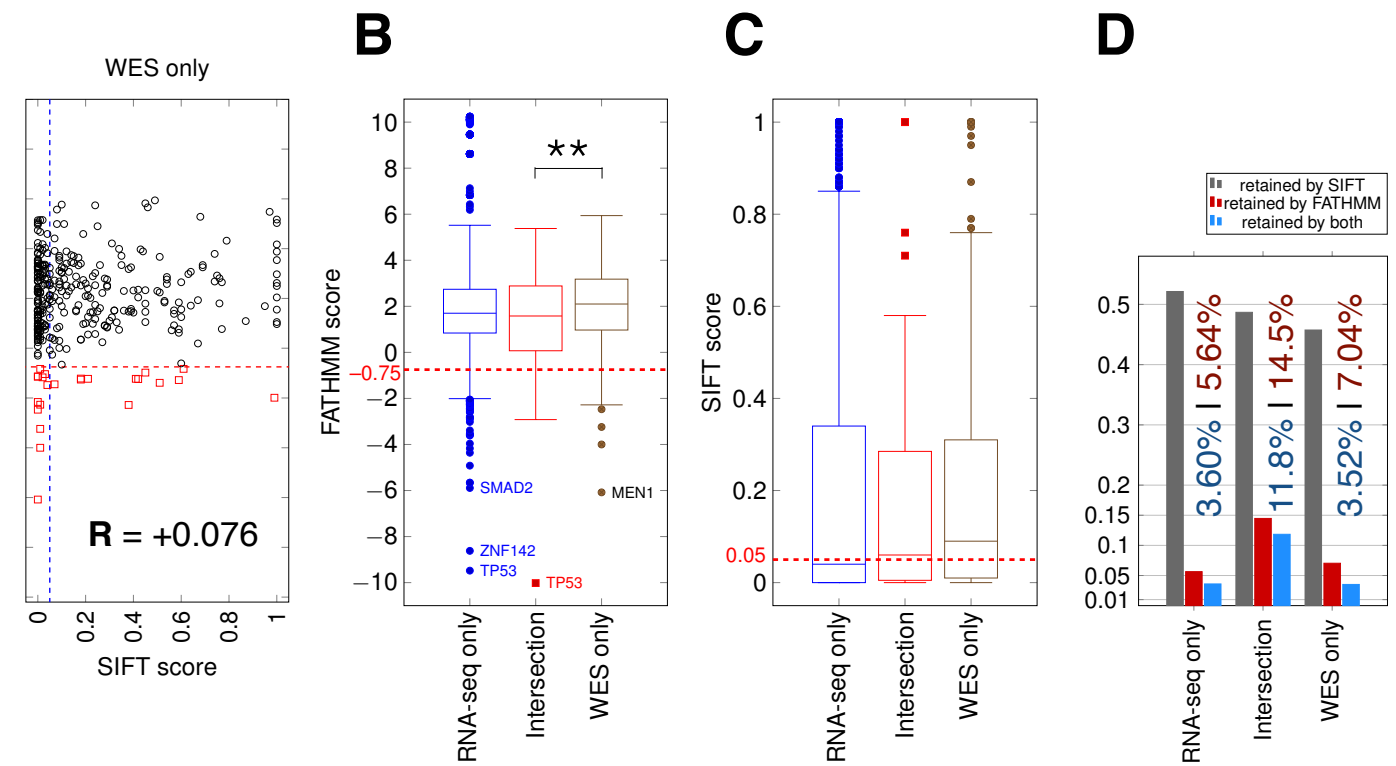


\section{Table $\mathbf{1}$ (on next page)}

"Best GBM-related mutations" from coding regions of SD01 and TCGA samples.

All variants shown were included in COSMIC and in a set of 29 GBM-related genes but not dbSNP. All variants are deleterious based on scoring by SIFT and FATHMM with cancer weights. For SIFTindel and FATHMM indels, the score is given as a confidence score of the prediction. AF (Allele Fraction, tumor) shows the proportion of altered reads in tumor samples, with Coverage (tumor) being the total number of reads at the variant position. 


\begin{tabular}{|c|c|c|c|c|c|c|}
\hline Gene & Sample & $\begin{array}{l}\text { AA } \\
\text { change }\end{array}$ & $\begin{array}{l}\text { FATHMM } \\
\text { score }\end{array}$ & $\begin{array}{l}\text { SIFT } \\
\text { score }\end{array}$ & $\begin{array}{l}\text { AF } \\
\text { (Tumor) }\end{array}$ & $\begin{array}{l}\text { Coverage } \\
\text { (Tumor) }\end{array}$ \\
\hline EGFR & $\begin{array}{l}\text { SD01 RNA-seq } \\
\text { only }\end{array}$ & A702S & $\begin{array}{l}-0.97 \\
(\mathrm{CANCER})\end{array}$ & 0.01 (Del) & 0.015 & 852 \\
\hline EGFR & SD01 Intersection & A289V & $\begin{array}{l}-1.04 \\
(\mathrm{CANCER})\end{array}$ & $\begin{array}{l}0.002 \\
(\mathrm{Del})\end{array}$ & 0.072 & 125 \\
\hline EGFR & $\begin{array}{l}\text { GBM01 } \\
\text { Intersection }\end{array}$ & G63R & $\begin{array}{l}-1.93 \\
(\mathrm{CANCER})\end{array}$ & $0.0(\mathrm{Del})$ & 0.175 & 296 \\
\hline TP53 & $\begin{array}{l}\text { GBM01 } \\
\text { Intersection }\end{array}$ & G105R & $\begin{array}{l}-10.02 \\
(\mathrm{CANCER})\end{array}$ & 0.0 (Del) & 0.44 & 50 \\
\hline TP53 & $\begin{array}{l}\text { GBM02 RNA- } \\
\text { seq only }\end{array}$ & $\mathrm{I} 254 \mathrm{~S}$ & $\begin{array}{l}-9.48 \\
(\mathrm{CANCER})\end{array}$ & 0.0 (Del) & 0.949 & 390 \\
\hline $\mathrm{TSC} 2$ & $\begin{array}{l}\text { GBM02 RNA- } \\
\text { seq only }\end{array}$ & V296fs & $\begin{array}{l}71 \% \\
\text { (pathogenic) }\end{array}$ & $\begin{array}{l}85.8 \% \\
(\mathrm{Dam})\end{array}$ & 0.137 & 55 \\
\hline PTEN & $\begin{array}{l}\text { GBM02 } \\
\text { Intersection }\end{array}$ & D107Y & $\begin{array}{l}-3.06 \\
(\mathrm{CANCER})\end{array}$ & $0.0(\mathrm{Del})$ & 0.69 & 92 \\
\hline PTEN & $\begin{array}{l}\text { GBM03 } \\
\text { Intersection }\end{array}$ & R173H & $\begin{array}{l}-6.42 \\
(\mathrm{CANCER})\end{array}$ & $0.0(\mathrm{Del})$ & 0.331 & 173 \\
\hline PTEN & $\begin{array}{l}\text { GBM04 } \\
\text { Intersection }\end{array}$ & D326fs & $\begin{array}{l}88 \% \\
\text { (pathogenic) }\end{array}$ & $\begin{array}{l}85.8 \% \\
(\mathrm{Dam})\end{array}$ & 0.393 & 146 \\
\hline PTEN & $\begin{array}{l}\text { GBM07 WXS } \\
\text { only }\end{array}$ & R130Q & $\begin{array}{l}-5.84 \\
(\mathrm{CANCER})\end{array}$ & $0.0(\mathrm{Del})$ & 0.713 & 190 \\
\hline NF1 & $\begin{array}{l}\text { GBM10 WXS } \\
\text { only }\end{array}$ & $\mathrm{C} 622 \mathrm{~F}$ & $\begin{array}{l}-0.83 \\
(\mathrm{CANCER})\end{array}$ & 0.01 (Del) & 0.403 & 389 \\
\hline
\end{tabular}




\section{Table 2 (on next page)}

Variants unknown by both COSMIC and dbSNP and candidates to be new GBM-related functional somatic mutations.

Variants included in the set of 29 GBM-related genes and not included in COSMIC or dbSNP are shown, although COSMIC contained alternative variants at the same positions for four mutations that were found by RNA-seq-only. For SIFTindel and FATHMM indels, the score is given as a confidence score. AF (tumor) shows the proportion of altered reads in tumor samples, with Coverage (tumor) being the total number of reads at the variant position. Allele Fraction and Coverage was used to further exclude potential artifacts, which are not listed here. 


\begin{tabular}{|c|c|c|c|c|c|c|c|}
\hline Gene & Sample & $\begin{array}{l}\text { AA } \\
\text { change }\end{array}$ & $\begin{array}{l}\text { FATHMM } \\
\text { score }\end{array}$ & $\begin{array}{l}\text { SIFT } \\
\text { score }\end{array}$ & COSMIC & $\begin{array}{l}\text { AF } \\
\text { (Tumor) }\end{array}$ & $\begin{array}{l}\text { Coverage } \\
\text { (Tumor) }\end{array}$ \\
\hline EGFR & $\begin{array}{l}\text { SD01 } \\
\text { RNA-seq- } \\
\text { only }\end{array}$ & S229fs & $\begin{array}{l}93 \% \\
\text { (pathogenic) }\end{array}$ & $\begin{array}{l}85.8 \% \\
\text { (Dam) }\end{array}$ & $\begin{array}{l}\text { no } \\
\text { (S229C) }\end{array}$ & 0.045 & 169 \\
\hline EGFR & $\begin{array}{l}\text { SD01 } \\
\text { RNA-seq- } \\
\text { only }\end{array}$ & W477fs & $51 \%$ (neutral) & $\begin{array}{l}85.8 \% \\
\text { (Dam) }\end{array}$ & $\begin{array}{l}\text { no } \\
\left(\mathrm{W} 477^{*}\right)\end{array}$ & 0.046 & 447 \\
\hline PIK3C2 & $\begin{array}{l}\text { SD01 } \\
\text { WES-only }\end{array}$ & $\mathrm{I} 255 \mathrm{~N}$ & $\begin{array}{l}-3.49 \\
(\mathrm{CANCER})\end{array}$ & 0 (Del) & no & 0.433 & 64 \\
\hline CDKN2C & $\begin{array}{l}\text { GBM02 } \\
\text { RNA-seq- } \\
\text { only }\end{array}$ & V130A & $\begin{array}{l}-0.21 \\
\text { (PASSENGER) }\end{array}$ & $\begin{array}{l}0.03 \\
(\mathrm{Del})\end{array}$ & no & 0.027 & 470 \\
\hline PDGFRB & $\begin{array}{l}\text { GBM02 } \\
\text { RNA-seq- } \\
\text { only }\end{array}$ & V840A & $\begin{array}{l}-2.34 \\
(\mathrm{CANCER})\end{array}$ & $\begin{array}{l}0.23 \\
(\text { Tol })\end{array}$ & no & 0.021 & 262 \\
\hline RB1 & $\begin{array}{l}\text { GBM03 } \\
\text { RNA-seq- } \\
\text { only }\end{array}$ & L872fs & $\begin{array}{l}77 \% \\
\text { (pathogenic) }\end{array}$ & $\begin{array}{l}85.8 \% \\
(\text { Dam })\end{array}$ & no & 0.035 & 355 \\
\hline EGFR & $\begin{array}{l}\text { GBM05 } \\
\text { RNA-seq- } \\
\text { only }\end{array}$ & M600T & $\begin{array}{l}-1.69 \\
(\mathrm{CANCER})\end{array}$ & $\begin{array}{l}0.38 \\
(\mathrm{Tol})\end{array}$ & $\begin{array}{l}\text { no } \\
(\mathrm{M} 600 \mathrm{~V})\end{array}$ & 8.1E-03 & 6240 \\
\hline EGFR & $\begin{array}{l}\text { GBM05 } \\
\text { RNA-seq- } \\
\text { only }\end{array}$ & L718R & $\begin{array}{l}-2.85 \\
(\mathrm{CANCER})\end{array}$ & 0 (Del) & $\begin{array}{l}\text { no } \\
\text { (L718M) }\end{array}$ & $4.5 \mathrm{E}-03$ & 4792 \\
\hline PDGFRB & $\begin{array}{l}\text { GBM06 } \\
\text { RNA-seq- } \\
\text { only }\end{array}$ & Q1075R & $\begin{array}{l}-1.25 \\
(\mathrm{CANCER})\end{array}$ & $\begin{array}{l}0.52 \\
(\mathrm{Tol})\end{array}$ & no & 0.058 & 90 \\
\hline
\end{tabular}

